\title{
IRRADIATION EFFECTS ON COMETS AND COMETARY DEBRIS
}

\author{
G. STRAZZULLA \\ Istituto di Astronomia \\ Citta' Universitaria \\ Viale A. Doria 6 \\ I-95125 Catania \\ Italy
}

\author{
R.E. JOHNSON \\ Dept. of Engineering and \\ Engineering Physics \\ University of Virginia \\ Charlottesville, VA 22901 \\ USA
}

\begin{abstract}
For the last 10 years, many experimental results have been obtained on the chemical and physical changes induced by ion and electron irradiation of materials relevant to comets. Those results are reviewed here, together with their physical interpretation and their relevance for cometary astrophysics. Cometary material is, from the time of its origin, altered by the large amount of energy deposited by energetic ions. Four phases of the irradiation history are considered: the pre-cometary phase, during which interstellar dust is bombarded by cosmic-ray ions; the accretion phase, during which comets are built up, possibly in an environment rich in fast ions (T-Tau phase); the cometary phase, during which the outer layers of the comets are irradiated by galactic ions in the Oort cloud; and the post-cometary phase, during which dust, lost from the comet, is bombarded by solar ions.

The relevant applications of laboratory results are reviewed. In particular, the ability of ion irradiation of simple carbon-containing ices to produce complex refractory organic materials is discussed. In the Oort cloud, this process can occur several meters into the surface, so that the buildup of a stable organic crust may occur. A comparison of ion irradiation at various stages is also made with other models for the production of organics.
\end{abstract}

\section{When, Where, and How Much}

Extended bodies in the Solar System (SS) were made by aggregation of smaller components. In the very early SS, grains accreted to form comets, presumably at a distance $>50 \mathrm{AU}$ from the Sun. These comets were then dynamically expelled to a distance of $10^{4}$ to $10^{5} \mathrm{AU}$ (the Oort cloud), where about $10^{12}$ comets have been stored for $4.6 \times 10^{9} \mathrm{yr}$. (Alternatively, comets could have been formed in situ at distances of a few tenths of a parsec from the Sun.) As we will discuss in detail in the following, it is possible that the pre-cometary grains were not formed directly in the primitive solar nebula, but were already present as interstellar grains in the interstellar cloud whose collapse gave rise to our SS. In other words, pre-cometary grains could have been formed and processed in the interstellar cloud and, therefore, may be already as old as $>10^{9} \mathrm{yr}$ before their aggre- 
gation in the SS. (Note: The lifetime of an interstellar molecular cloud has been estimated as $3 \times 10^{7}$ to $5 \times 10^{8}$ yr or more (e.g., Greenberg 1982). It is, however, likely that a pre-cometary grain passes many times $(\simeq 20)$ from a diffuse cloud to a dense one before the cloud condenses to form a sun.) When comets are ejected from the Oort cloud and visit the inner SS, these grains are ejected and/or evaporated. They are believed to be important contributors of interplanetary grains, those that are collected in the upper atmosphere or those whose impact craters are counted on the Moon's surface.

During all of the stages described above, the cometary grains are subjected to physical and chemical processes that continuously modify their properties (evolution). Among these processes are the ones we discuss here, those induced by irradiation with energetic ( $\mathrm{keV}$ to $\mathrm{MeV}$ ) ions or electrons. Before discussing these effects, we trace the bombardment history of cometary material during the four stages sketched above.

The flux of low-energy cosmic rays irradiating grains in the interstellar medium is not well-known. De Jong and Kamijo (1973) assumed a proton flux of J ( $E \cong$ $1 \mathrm{MeV})=36 \mathrm{~cm}^{-2} \mathrm{~s}^{-1}$, as postulated by Field et al. (1969), in order to produce an $\mathrm{H}$ atom ionization rate $\zeta_{\mathrm{H}}=4 \times 10^{-16} \mathrm{~s}^{-1}$. Some evidence indicates that $\zeta$ could be lower than this value (Watson 1975 and references therein). A proton flux of $J(E=1 \mathrm{MeV})=$ $10 \mathrm{~cm}^{-2} \mathrm{~s}^{-1}$ has been assumed by Strazzulla et al. (1983a). The range of a 1-MeV proton is on the order of $10^{-2}$ to $10^{-3} \mathrm{~cm}$ for organic materials with a density of $\rho=1 \mathrm{~g} / \mathrm{cm}^{3}$ (see the table on the ion range in all elements by Littmark and Ziegler 1980), i.e., much larger than a typical grain size $(0.1 \mu \mathrm{m})$. Thus, a cosmic-ray proton $(\mathrm{E}=1 \mathrm{MeV})$ passes through the grain, depositing part of its energy. If a pre-cometary grain resides for more than $10^{9} \mathrm{yr}$ in the interstellar medium, it is subjected to a total fluence (1-MeV protons) greater than $3 \times 10^{17} \mathrm{~cm}^{-2}$. The specific energy loss of a $1-\mathrm{MeV}$ proton in typical grain material containing heavy atoms $(\mathrm{C}, \mathrm{N}, \mathrm{O}$, and $\mathrm{Si})$ is on the order of $10^{-14} \mathrm{eV} \mathrm{cm}^{2} /$ atom. This implies an energy deposition of $10^{-14}(\mathrm{eV} \mathrm{cm} / \mathrm{mol}) \times 3 \times 10^{17}\left(\mathrm{~cm}^{-2}\right)=$ $3 \times 10^{3}(\mathrm{eV} / \mathrm{mol})$ in $10^{9} \mathrm{yr}$. Here and in the following, we will give the dose as $\mathrm{eV}$ per small molecule, e.g., $\mathrm{H}_{2} \mathrm{O}, \mathrm{NH}_{3}$, and $\mathrm{CH}_{4}$, as this is a convenient way to characterize chemical change. To apply this to molecules such as $\mathrm{CO}_{2}, \mathrm{CO}$, and $\mathrm{SiO}_{2}$, the dose must be scaled roughly according to molecular weight.

During the formation of the SS, the pre-cometary grains accreted to form larger and larger cometesimals. The details of the formation process are not known, nor is it clear if the pre-cometary grains are first destroyed and then reformed. In any case, it is obvious that fresh materials (frozen gases) would have to condense on refractory cores prior to the buildup of larger fragments. It is also plausible that this accretion process took place when the young Sun was very active, e.g., during the so-called T-Tau phase (Strazzulla 1985). The belief that the ancient Sun passed through a luminous stage (the T-Tau phase) has been based on the need to have a mechanism for the removal of excess mass by sweeping outward gas and dust left over by planetary aggregation. In recent years, increasing evidence has confirmed that there are gross similarities between present T-Tau stars and what we imagine the early SS was. The higher flux of photons and particles originating from such stars may be scaled to estimate the particle flux from the ancient Sun (Worden et al. 1981, Feigelson 1982). Strazzulla (1985) assumed a proton flux of $(\mathrm{E}>1 \mathrm{MeV}) \mathrm{J}=$ $10^{10} \mathrm{~cm}^{-2} \mathrm{~s}^{-1}$ at $1 \mathrm{AU}$ (varying as the inverse square of the solar distance) and $10^{6} \mathrm{yr}$ as duration of the T-Tau phase. If grains or very small cometesimals were irradiated (at $50 \mathrm{AU}$ from the Sun) by those particles, then they suffered a total dose of $\phi>10^{20} \mathrm{p}$ $(>1 \mathrm{MeV}) / \mathrm{cm}^{2}$ or about $10^{6} \mathrm{eV} / \mathrm{mol}$. 
Once formed and expelled from the outer SS into the Oort cloud, comets have been exposed for about $4.6 \times 10^{9} \mathrm{yr}$ to the flux of galactic cosmic rays. Leger et al. (1985) used for galactic protons:

$$
\begin{array}{ll}
\mathrm{J}_{\mathrm{H}}(\mathrm{E})=0.3 \mathrm{E}^{-1} & 0.2<\mathrm{E}<1 \\
\mathrm{~J}_{\mathrm{H}}(\mathrm{E})=0.3 \mathrm{E}^{-2} & 1<\mathrm{E}
\end{array}
$$

where $\mathrm{J}$ is given in protons $\mathrm{cm}^{-2} \mathrm{sr}^{-1} \mathrm{~s}^{-1} \mathrm{GeV}^{-1}$ and $\mathrm{E}$ is in GeV. Johnson et al. (1987) estimated that the external $(0.1$ to $0.5 \mathrm{~m})$ layers of a comet were subjected to an irradiation dose of $600 \mathrm{eV} / \mathrm{mol}$. Deeper layers were subjected to a lower dose because the most abundant but less energetic ions are stopped by the external layers.

If the formation of comets occurred within $10^{6} \mathrm{yr}$ after the production of the radioactive isotope ${ }^{26} \mathrm{Al}$, then the cometary nucleus has been uniformly irradiated, due to the decay products of that isotope. The absorbed dose has been estimated to be about $3 \mathrm{eV} / \mathrm{mol}$ (Draganic at al. 1984). Once a comet penetrates the inner SS (<2 AU) and the coma is developed, large quantities of cometary materials are lost. It is thought that they constitute a large fraction of those interplanetary grains (interplanetary dust particles [IDPs]) that produce the zodiacal light or that have been collected and are known as Brownlee particles. Theoretical considerations and, recently, the observation of ion tracks in the silicate IDPs (Bradley et al. 1984) support the idea that IDPs stay for $10^{4}$ to $10^{5} \mathrm{yr}$ in the interplanetary medium before arrival and collection at Earth. During this time, these micrometer-sized particles are subjected to the flux of solar ions (Strazzulla et al. 1985, Johnson 1985, Johnson and Lanzerotti 1986). The flux of penetrating solar protons $(E=100 \mathrm{keV})$ has been evaluated at about $10^{11} \mathrm{~cm}^{-2} \mathrm{yr}^{-1}$ at $1 \mathrm{AU}$. This is equivalent to a total dose on the order of 10 to $100 \mathrm{eV} / \mathrm{mol}$. The flux of solar wind particles provides a much larger dose ( $\left.\cong 10^{5} \mathrm{eV} / \mathrm{mol}\right)$, but only to a depth of $\cong 0.02 \mu \mathrm{m}$.

The fluences suffered by cometary material during the four stages of its life are summarized in Table 1. The very high net dose of energy absorbed per exposed molecule indicates clearly that the study of the interaction of energetic ions with cometary material is of fundamental relevance to understanding the evolution of comets. In recent years, these studies have been conducted mainly by performing laboratory simulations and bombarding relevant targets with charged particles at physical conditions more or less similar to the astrophysical ones.

\section{Laboratory Simulations}

In laboratory simulations of irradiation of cometary materials, there are several parameters to be reproduced. Among these are the mass and energy of the incoming ion, total implanted dose, fluence (number of particles per unit area), dose rate (or flux), and composition and physical state of the target.

\subsection{ION MASS AND ENERGY}

By using electrostatic accelerators, it is possible to obtain any kind of ion. Thus, the mass is not a difficult parameter to reproduce. However, most of the experiments are 
Table 1. Irradiation history of an ideal cometary grain $\left(\rho=1 \mathrm{~g} / \mathrm{cm}^{3} ; \mathrm{a}=0.1 \mu \mathrm{m}\right)$. (Note: Particles of radius $0.1 \mu \mathrm{m}$ are typical of interstellar and IDP grains. It is relevant that grains have diameters smaller than the range of considered ions.)

\begin{tabular}{|c|c|c|c|}
\hline $\begin{array}{l}\text { Time } \\
(\mathrm{yr})\end{array}$ & Status & $\begin{array}{c}\text { Total Fluence } \\
\left(\mathrm{MeV} \text { protons } / \mathrm{cm}^{2}\right)\end{array}$ & $\begin{array}{l}\text { Total Dose } \\
(\mathrm{eV} / \mathrm{mol})\end{array}$ \\
\hline$\simeq 10^{9}$ & Interstellar grain & $3 \times 10^{17}$ & $3 \times 10^{3}$ \\
\hline$\simeq 10^{6}$ & $\begin{array}{l}\text { Pre-cometary grain } \\
\text { (T-Tau phase) }\end{array}$ & $10^{20}$ (at $50 \mathrm{AU}$ ) & $10^{6}$ \\
\hline $4.6 \times 10^{9}$ & Comet & $\begin{array}{l}\mathrm{J}=0.3 \mathrm{E}^{-1} \quad(0.2<\mathrm{E}<1) \\
\mathrm{J}=0.3 \mathrm{E}^{-2} \quad(\mathrm{E}>1) \\
\mathrm{p} \mathrm{cm} \mathrm{cm}^{-2} \mathrm{sr}^{-1} \mathrm{~s}^{-1} \mathrm{GeV}^{-1}(\mathrm{E} \text { in } \mathrm{GeV})\end{array}$ & $\begin{array}{l}6 \times 10^{2} \\
(\mathrm{at} \simeq 0.1 \mathrm{~m})\end{array}$ \\
\hline $10^{4}-10_{5}$ & IDP & $\begin{array}{l}10^{15}-10^{16}(\mathrm{E} \cong 100 \mathrm{keV}) \\
10^{19}-10^{20}(\mathrm{E} \cong 1 \mathrm{keV})\end{array}$ & $\begin{array}{l}10-100 \\
10^{5}-10^{6}\end{array}$ \\
\hline
\end{tabular}

made with light ions because, except in some specific circumstances, light ions are most abundant and penetrate into the material more deeply.

It is very difficult to reproduce the energy spectrum of, e.g., galactic protons (Eq. 1). However, the physico-chemical solid-state effects we discuss here are often attributable to ions whose energy vary from a few $\mathrm{keV}$ to a few $\mathrm{MeV}$. These energies are easily obtained in the laboratory and, when necessary, the results have been theoretically extrapolated to different energy ranges.

\subsection{TOTAL IMPLANTED DOSE AND DOSE RATE}

To reproduce the total ion fluence (see column 3 in Table 1) is not a difficult task. However, any experimental result that should depend on the total implanted dose (e.g., effects of charge accumulation) cannot be directly applied without noting that the same accumulation has occurred on a very different time scale in astrophysics. One has to be sure that, during the accumulation time, other processes (e.g., resurfacing, diffusion, or charge neutralization) have not altered the target. It is, of course, impossible to reproduce the dose rate and obtain measurable effects. The laboratory experiments can be performed at dose rates not lower than $\cong 0.01$ to $0.1 \mu \mathrm{A} \cong 0.6$ to $6 \times 10^{11}$ ions $\mathrm{cm}^{-2} \mathrm{~s}^{-1}$. These rates are orders of magnitude greater than in astrophysics. Therefore, any experimental result that depends on the dose rate, as, for example, beam-induced heating of the target, cannot be extrapolated to astrophysical scenarios. 


\subsection{COMPOSITION AND PHYSICAL STATE OF THE TARGET}

Although many materials can be studied, the astrophysical composition is surely the most difficult thing to reproduce, because if one knew the exact composition and physical state of the cometary material, then the simulation experiments would not be necessary! Since we do not know the exact composition and physical state of a comet, we cannot simulate a cometary target. Therefore, we do not call the experiments "simulation experiments." However, what is extremely important is that we can measure some parameters that, under specific circumstances, are useful for predicting effects occurring in astrophysics. When a nuclear physicist measures a cross section for a nuclear process, he does not attempt to simulate the interior of a star; nevertheless, the measurement of the cross section is crucial for understanding stellar evolution. Analogously, the measurement of, for example, sputtering yields or cross sections for the alteration of simple molecular solids does not simulate the alteration of a grain or a comet, but it is needed for understanding the evolution of cometary materials. As cometary models suggest which kinds of targets or processes are most useful to study, a sketch of relevant models is given in the next section.

\section{Cometary Materials}

Before Halley's recent passage, the accepted cometary model was Whipple's (1950) dirty snowball model. According to this model, comets are made of a mixture of refractory materials and ices. Since that time, a big debate has developed about the structure of comets and about the complexity of the cometary materials.

In recent years, other cometary models have been developed that are essentially variations of Whipple's model, e.g., the interstellar dust model (Greenberg 1982), the fractal model (Donn and Hughes 1986), the icy-glue model (Gombosi and Houpis 1986), and the rubble model (Weissman 1986). Although such models attempt to explain many structural characteristics and approximate composition, none have been tested in any detail. However, what is important for the purpose of the present review is that all of these models agree that simple molecular ices such as $\mathrm{H}_{2} \mathrm{O}, \mathrm{CO}, \mathrm{CO}_{2}, \mathrm{NH}_{3}$, and $\mathrm{CH}_{4}$ are abundant constituents of a comet. Since this has been confirmed by observations and since simple molecules have been identified in the interstellar medium, it makes sense to study the effect of irradiation on these materials.

In addition, before the spacecraft encounters, the presence of more or less complex organic (or even biological) molecules was suggested on theoretical grounds (Vanysek and Wickramasinghe 1975, Hoyle and Wickramasinghe 1985), on the basis of laboratory experiments (Greenberg 1982, Strazzulla 1986) and telescopic observations (Hanner et al. 1985). The presence of such materials has been emphatically confirmed by spacecraft observations. Therefore, irradiation experiments on a variety of more or less complex organic materials have been carried out.

\section{Experimental Procedure and Results}

Ion irradiation experiments have been performed on a variety of astrophysically relevant materials, including: 
(1) Molecular solids (e.g., frozen $\mathrm{H}_{2} \mathrm{O}, \mathrm{CO}_{2}, \mathrm{CO}, \mathrm{NH}_{3}, \mathrm{SO}_{2}, \mathrm{~S}$, and various mixtures).

(2) Hydrocarbons $\left(\mathrm{C}_{6} \mathrm{H}_{6}, \mathrm{C}_{6} \mathrm{D}_{6}, \mathrm{CH}_{4}\right.$, etc.).

(3) Aliphatic and aromatic polymers (e.g., polyethylene, polyimide, polystyrene, polypropylene, and Teflon).

(4) Complex molecules (e.g., 1,4 diaminobutane).

(5) Biological materials (e.g., melanin, and bacillus subtilis).

(6) Refractory solids (silicates, etc.).

The quantities that have been measured include:

(1) Sputtering yields (the number of atoms or molecules released per impinging ion).

(2) Type and amount of (mainly neutral) molecules released during the bombardment (sputtering) phase or during the warm-up after irradiation.

(3) Alteration of the stoichiometry (atomic composition) of the bombarded target.

(4) Production of refractory organic residues.

(5) Alterations of the IR absorption spectra.

(6) Changes in the structure and reflectance

(7) Variations in physical quantities (e.g., density).

The experimental procedure includes the preparation of targets in a clean vacuum, the "in situ" bombardment by the chosen ions at a given energy and for a specified fluence, the "in situ" analysis of the target before and during the experiment, and, occasionally, the warm-up of the irradiated sample and laboratory analysis of the residue.

The preparation of the target depends on its initial physical state (solid, liquid, or gas). Solids are simply put on a sample holder whose temperature can be changed between 10 and $400 \mathrm{~K}$. Volatile species are condensed on a previously cooled substratum by admitting vapors into the scattering chamber through a needle valve. The vacuum in the various scattering chambers used varies between $10^{-10}$ and $10^{-4}$ mbar. A schematic view of an experimental apparatus is shown in Figure 1, where D indicates one of the many possible detectors. Ion beams ( $\mathrm{H}, \mathrm{He}$, and heavier ions) are generally obtained from a $\mathrm{MeV}$ Van de Graaff accelerator or a keV ion implanter at energies between about $1 \mathrm{keV}$ and a few MeV. Low-energy accelerators are also used occasionally. Ion currents between a few $\mathrm{nA} / \mathrm{cm}^{2}$ and $100 \mu \mathrm{A} / \mathrm{cm}^{2}$ and ion fluences in the range of $10^{12}$ to $10^{19}$ ions $/ \mathrm{cm}^{2}$ have been used.

The thickness (atoms $/ \mathrm{cm}^{2}$ ) of the deposited layer is commonly measured "in situ." The variation of the thickness with ion fluence is used to obtain the erosion yield. The thickness is measured using techniques such as alpha-particle energy loss (O'Shaughnessy et al. 1986), Rutherford Backscattering (RBS) (e.g., Brown et al. 1980), and Proton Induced X-ray Emission (PIXE) (e.g., Torrisi et al. 1986). These techniques can also give information on the morphological properties of the irradiated sample (flat versus rough surfaces), and the last two (RBS and PIXE) can give information on the atomic composition of the target as well. During irradiation, products are ejected from the target. These products include atoms, molecules, backscattered ions, electrons, and photons. Observing 


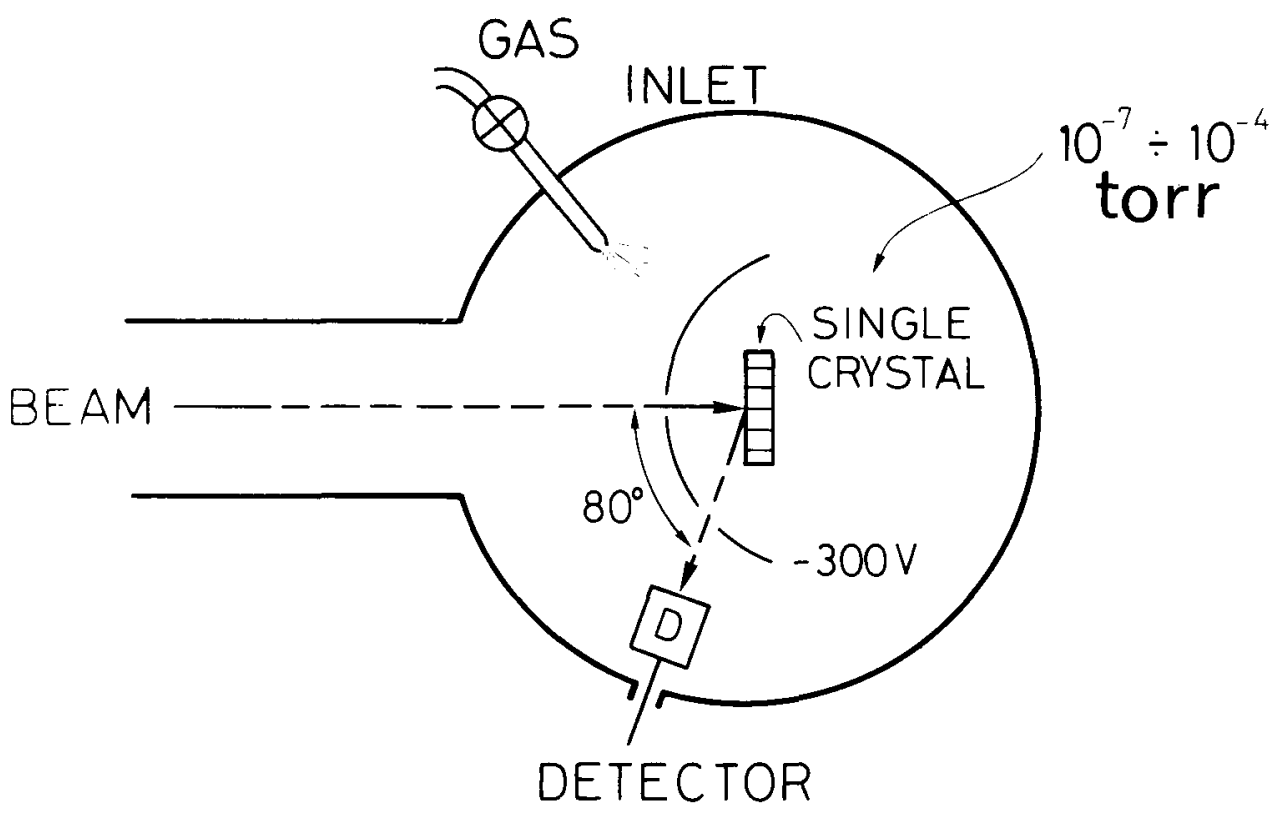

Figure 1. Schematic view of the experimental apparatus. (D) indicates one of the many possible detectors.

these products gives essential information on the material changes induced by the bombarding ions. Thus, techniques such as neutral mass spectrometry and the detection of X-rays, backscattered ions, and light have been used. Optical properties have been investigated by illuminating, through opportune windows, the bombarded target and detecting the radiation reflected by the surface or transmitted through the sample.

Once bombarded, the target can be warmed to room temperature while the material loss is observed. Then this material is extracted from the chamber. If a refractory material was used or is produced, as, for example, after the irradiation of carbon-containing frosts (the well-known organic residue), then it can be analyzed by other laboratory techniques (SEM, TEM, ESCA, REELS, or Raman), and such things as the optical (UV-Vis-IR) properties and physical parameters (e.g., density) can be studied.

In Table 2, the experimental values of the erosion yield obtained by bombarding low-temperature samples with $1.5-\mathrm{MeV} \mathrm{He}$ ions are reported, together with the target temperature at which they have been obtained and the most abundant neutral species released during erosion. The yields reported in Table 2 refer to a fresh layer of material. As chemical changes occur during irradiation, the target is altered and it can become sputter-resistant. This has been observed for carbon-containing (Lanzerotti et al. 1985, Foti et al. 1987, Chrisey et al. 1989) and sulfur-containing targets (Melcher et al. 1982, Boring et al. 1985, Torrisi et al. 1988). It is interesting to note that ion irradiation causes the formation of both more volatile species and less volatile ones. The most volatile species formed along the ion 
Table 2. Erosion yields by $1.5-\mathrm{MeV}$ He ions of selected targets are given together with the most abundant released neutral species. For the $(\mathrm{dE} / \mathrm{dx})$ dependence, see Johnson et al. (1984).

\begin{tabular}{cccc}
\hline Target & $\begin{array}{c}\text { Erosion Yield } \\
\text { (mol/1.5-MeV He ion) }\end{array}$ & $\mathrm{T}(\mathrm{K})$ & Most Abundant \\
$\mathrm{H}_{2} \mathrm{O}$ & 8 & $10-77$ & $\mathrm{H}_{2}, \mathrm{O}_{2}, \mathrm{H}_{2} \mathrm{O}$ \\
$\mathrm{CO}_{2}$ & 120 & 10 & $\mathrm{CO}, \mathrm{CO}_{2}, \mathrm{O}_{2}$ \\
$\mathrm{CH}_{4}$ & 100 & 10 & $\mathrm{H}_{2}, \mathrm{C}_{2} \mathrm{H}_{2}, \mathrm{CH}_{4}$ \\
$\mathrm{NH}_{3}$ & 140 & 10 & $\mathrm{H}_{2}, \mathrm{~N}_{2}, \mathrm{NH}_{3}, \mathrm{~N}_{2} \mathrm{H}_{4}$ \\
$\mathrm{SO}_{2}$ & 16 & $10-77$ & $\mathrm{O}_{2}, \mathrm{SO}, \mathrm{SO}_{2}, \mathrm{SO}_{3}$ \\
$\mathrm{SS}_{8}(\mathrm{a})$ & 10 & $77-200$ & $\mathrm{~S}_{2}$ \\
\hline
\end{tabular}

(a) Generally initially deposited as $\mathrm{S}_{8}$, but upon erosion, it is fragmented and hence changes color (Chrisey et al. 1988).

tracks may diffuse outwards and may be eventually expelled from the target (see the last column in Table 2).

Complex molecules can also be formed efficiently when mixtures are irradiated, but because of their lower volatility, they are primarily released upon warming up of the target; they can also be ejected when an heavy energetic ion strikes the sample.

For instance, Pirronello et al. (1982) noted the formation of $\mathrm{H}_{2} \mathrm{CO}$ by irradiating a mixture of $\mathrm{H}_{2} \mathrm{O}+\mathrm{CO}_{2}$ with 1.5-MeV He ions; Moore et al. (1983) irradiated various mixtures of $\mathrm{H}_{2} \mathrm{O}, \mathrm{NH}_{3}, \mathrm{CH}_{4}, \mathrm{~N}_{2}, \mathrm{C}_{3} \mathrm{H}_{8}, \mathrm{CO}$, and $\mathrm{CO}_{2}$ with $\sim \mathrm{MeV}$ protons and reported the synthesis of $\mathrm{C}_{2} \mathrm{H}_{6}, \mathrm{~N}_{2} \mathrm{O}, \mathrm{NO}, \mathrm{C}_{2} \mathrm{H}_{4}$, and $\mathrm{C}_{3} \mathrm{H}_{8}$; and Haring et al. (1983) bombarded $\mathrm{H}_{2} \mathrm{O}+\mathrm{NH}_{3}+\mathrm{CO}$ with $\mathrm{keV}$ ions and reported the formation of $\mathrm{H}_{2} \mathrm{CO}$ and $\mathrm{N}_{2} \mathrm{H}_{4}$.

Similar products may be produced by UV photons.

To exemplify the difference in the erosion of $\mathrm{H}_{2} \mathrm{O}$ and $\mathrm{CH}_{4}$, two targets of astrophysical interest, the variations of thickness and of the ratio $\mathrm{O} / \mathrm{H}$ (or $\mathrm{C} / \mathrm{H}$, respectively) are shown in Figure 2 versus ion fluence. It is clearly seen that, during irradiation, the behavior of the two frozen targets is completely different.

For water, ion-induced ejection of $\mathrm{H}_{2} \mathrm{O}, \mathrm{H}_{2}$, and $\mathrm{O}_{2}$ has been observed (Brown et al. 1982b). Although $\mathrm{H}_{2}$ and $\mathrm{O}_{2}$ exhibit initial large and transient ejection (Reimann et al. 1984), their ejection rate is much smaller (about 10\%) than of $\mathrm{H}_{2} \mathrm{O}$ (at least at low $\mathrm{T}$ $[10 \mathrm{~K}]$, and for light ions). In addition, the $\mathrm{O} / \mathrm{H}$ ratio does not vary appreciably from the 


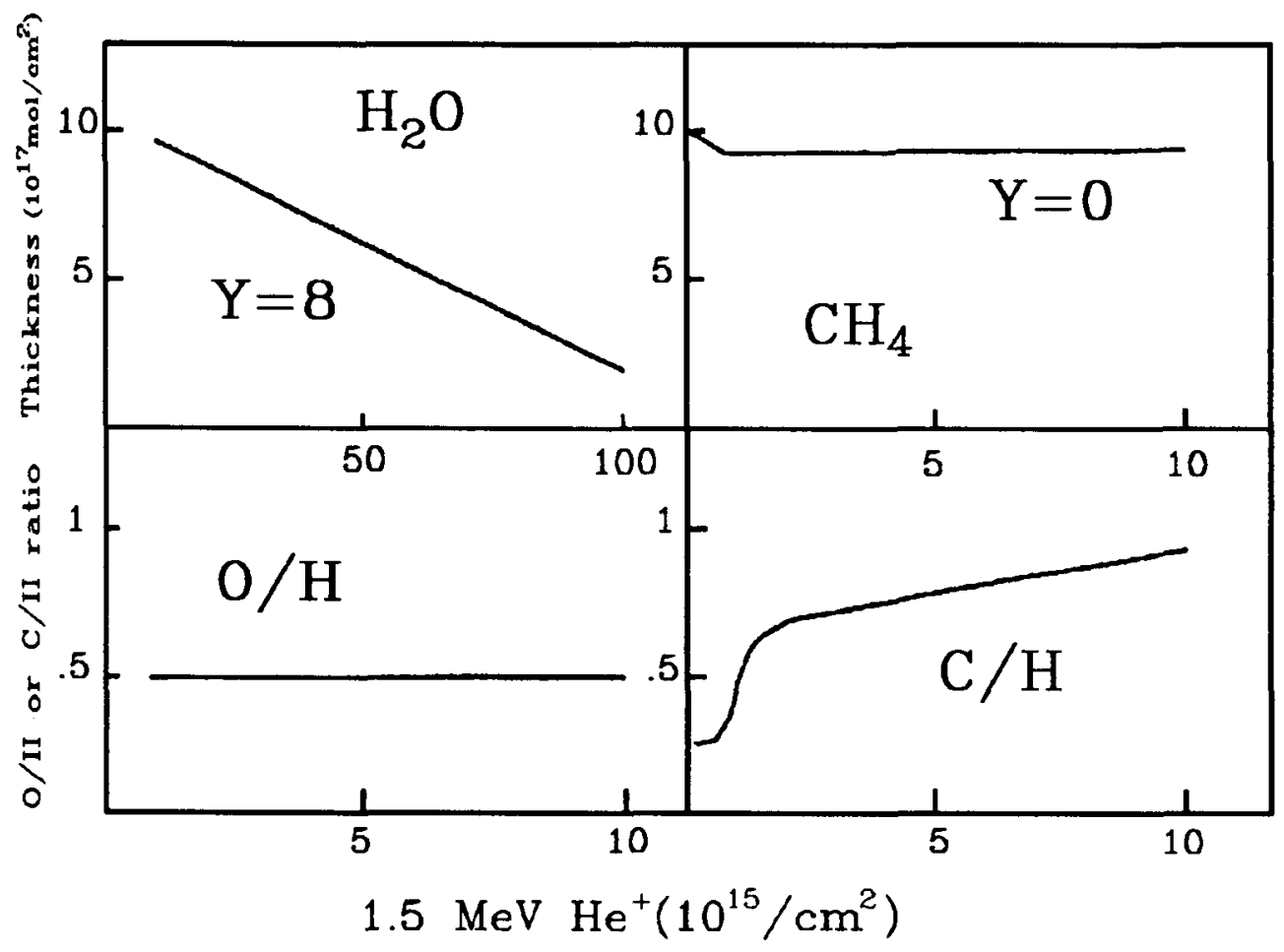

Figure 2. The variations of thickness and of the ratio $\mathrm{O} / \mathrm{H}$ and $\mathrm{C} / \mathrm{H}$ in irradiated frozen water and methane are shown versus ion $(1.5-\mathrm{MeV} \mathrm{He})$ fluence.

initial value of 0.5 . For methane, $\mathrm{H}_{2}$ is the most relevant emitted species (Brown et al. 1987), although at the beginning of irradiation, emission of $\mathrm{CH}_{4}$ and $\mathrm{C}_{n} \mathrm{H}_{m}$ has been observed (about $100 \mathrm{~mol} / 1.5-\mathrm{MeV} \mathrm{He}$ ion) (Foti et al. 1987, Lanzerotti et al. 1987). After a fluence equivalent to about $10^{14}$ ions $/ \mathrm{cm}^{2}(1.5-\mathrm{MeV} \mathrm{He})$, this yield becomes almost zero, due to the chemical change induced by the loss of $\mathrm{H}$.

The effective cross-section for the conversion of such volatile carbon-containing molecules to a refractory residue (polymerization) has been measured for a number of projectile-target combinations (Foti et al. 1984, Strazzulla et al. 1984). It has been found that the thickness $(\mathrm{T})$ of the residue $\left(\mathrm{C}\right.$-atoms $\left./ \mathrm{cm}^{2}\right)$ is approximately related to the initial thickness, $\mathrm{T}_{0}$, by:

$$
\mathrm{T} \simeq \mathrm{T}_{0}\left(1-\mathrm{e}^{-\sigma \phi}\right)
$$

where $\phi$ (ions $\left./ \mathrm{cm}^{2}\right)$ is the implanted dose, and $\sigma\left(\mathrm{cm}^{2}\right)$ the cross-section for the process that is independent of fluence at low fluences $\left(<10^{16}\right.$ ions $\left.\mathrm{cm}^{-2}\right)$. For methane irradiated by $\mathrm{MeV} \mathrm{H}$ ions, $\sigma$ varies linearly with the electronic stopping power at low fluence, as shown in Figure 3. 


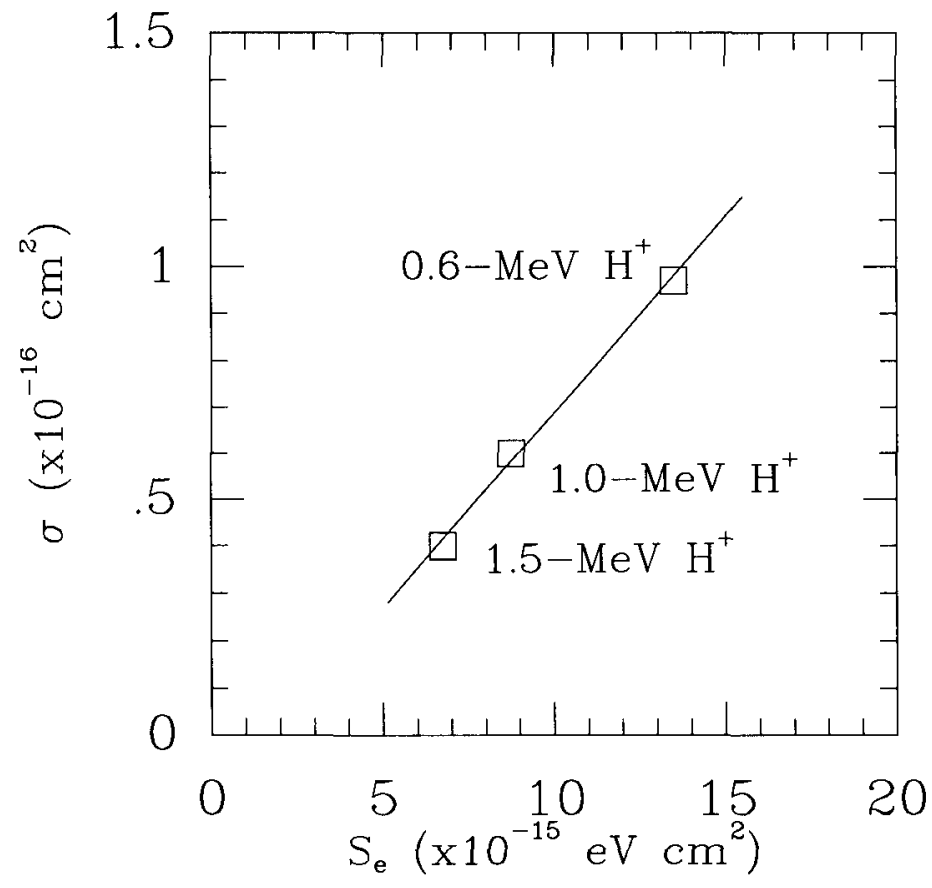

Figure 3. The cross-section $\sigma\left(\mathrm{cm}^{2}\right)$ for the process converting frozen methane to organic residue is shown versus the electronic stopping power of the incoming beam.

After extraction from the scattering chamber, the organic residue left over by ion irradiation can be analyzed (e.g., Moore and Donn 1982). In Figure 4, the IR spectra obtained from residues left over after $75-\mathrm{keV} \mathrm{H}$ ions bombardment of frozen methane at two different doses $\left(10^{16}\right.$ and $4.3 \times 10^{16}$ ions $\left./ \mathrm{cm}^{2}\right)$ are shown. The spectra are typical of polymer-like organic materials, with distinctive features around $2900 \mathrm{~cm}^{-1}(3.4 \mu \mathrm{m})\left(\mathrm{CH}_{2}\right.$ and $\mathrm{CH}_{3}$ stretching) and $1400 \mathrm{~cm}^{-1}(7.1 \mu \mathrm{m})\left(\mathrm{CH}_{2}\right.$ and $\mathrm{CH}_{3}$ bending). Two other prominent features are observed around $3450 \mathrm{~cm}^{-1}(2.9 \mu \mathrm{m})(\mathrm{O}-\mathrm{H}$ stretching $)$ and $1700 \mathrm{~cm}^{-1}(5.9 \mu \mathrm{m})(\mathrm{C}=\mathrm{O}$ and $\mathrm{C}=\mathrm{C}$ bonds $)$. These indicate the reactivity of the sample, which clearly trapped oxygen or water in the vacuum deposition process. From Figure 4, one can also see that the IR spectrum changes as the ion fluence increases. In particular, the strength of the IR bands due to $\mathrm{CH}$ bonds decreases because of $\mathrm{H}_{2}$ loss and carbonization.

The progressive carbonization of the irradiated target influences its optical properties in the UV-Vis range also, as has been noted in most laboratories. In particular, the slope of the reflectance spectra (4000 to $8000 \mathrm{~A}$ ) changes with the amount of energy deposited per molecule in the sample (Andronico et al. 1987). In Figure 5, spectra of three residues are plotted. Spectra labelled organic 1,2, and 3 refer, respectively, to residues obtained from frozen benzene irradiated by $100-\mathrm{keV} \mathrm{He}$ ions $\left(10^{15} / \mathrm{cm}^{2}\right.$, organic 1$)$, frozen 


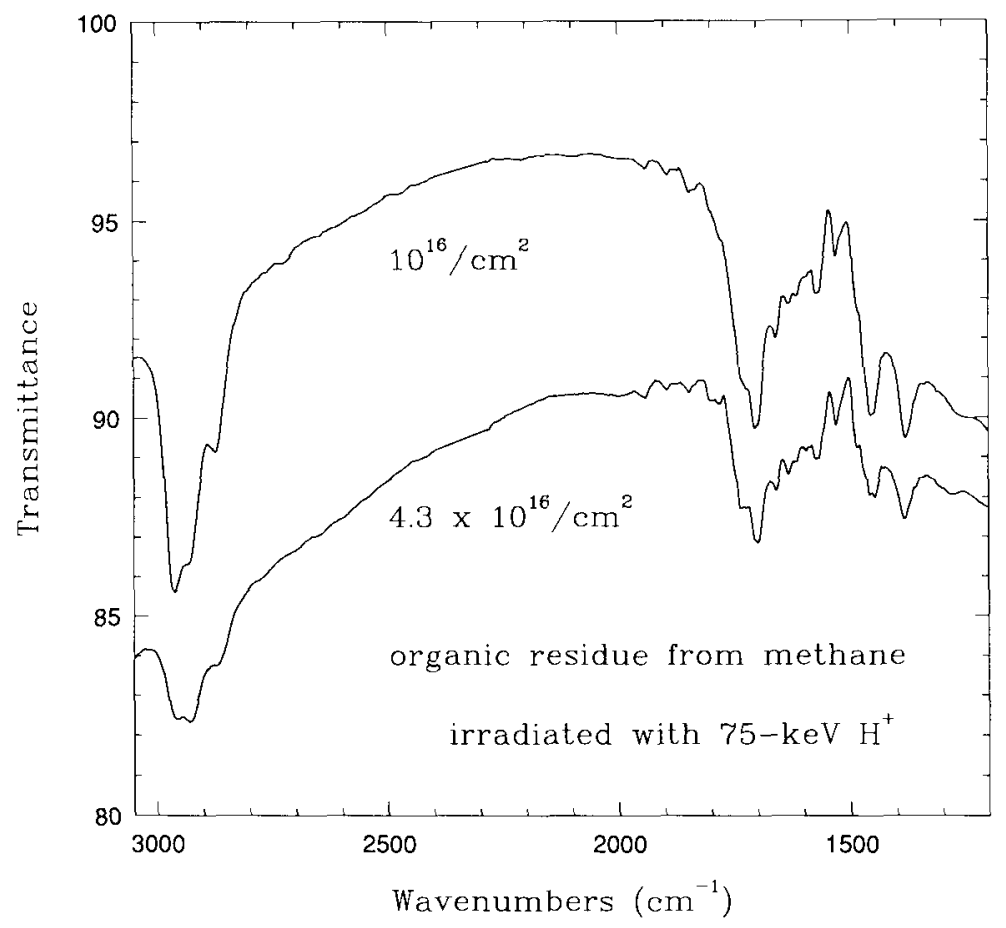

Figure 4. IR spectra of organic residue from ion-irradiated frozen methane.

benzene irradiated by $200-\mathrm{keV}$ Ar ions $\left(10^{15} / \mathrm{cm}^{2}\right.$, organic 2$)$, and methane irradiated by $1.5-\mathrm{MeV} \mathrm{H}$ ions $\left(10^{17} / \mathrm{cm}^{2}\right.$, organic 3$)$.

The density of the residue has been measured by comparing the thickness (Catoms $/ \mathrm{cm}^{2}$ ) measured in the scattering chamber with the physical thickness (Foti et al. 1984). In Figure 6, we see that the density of the organic residue as obtained from frozen methane after a fluence of $2 \times 10^{16} \mathrm{H}^{+} / \mathrm{cm}^{2}(1.5 \mathrm{MeV})$ is $0.5 \mathrm{~g} / \mathrm{cm}^{3}$, i.e., equal to that of the deposited methane. The residue appears very fluffy. Additional irradiation causes the density to increase as a result of further loss of hydrogen molecules and a compaction of the sample. This increase in density is also quite general and occurs for all frozen hydrocarbons and commercial polymers.

As an example of this, Figure 7 shows the variation of the density of polystyrene when bombarded by various ions both in the electronic (Figure 7a) and in the nuclear (Figure $7 \mathrm{~b}$ ) regime. We can see that in the electronic regime, the density increases from the initial $1 \mathrm{~g} / \mathrm{cm}^{3}$ to $\sim 1.25 \mathrm{~g} / \mathrm{cm}^{3}$ as the product of the fluence by the electronic stopping power (i.e., the energy deposited per C-atom) goes to $\sim 1.5 \times 10^{3} \mathrm{eV} /$ atom. More dramatic is the increase observed in the nuclear regime, with the density reaching $1.75 \mathrm{~g} / \mathrm{cm}^{3}$ after an energy deposition of $3 \times 10^{2} \mathrm{eV} / \mathrm{C}$-atom. 


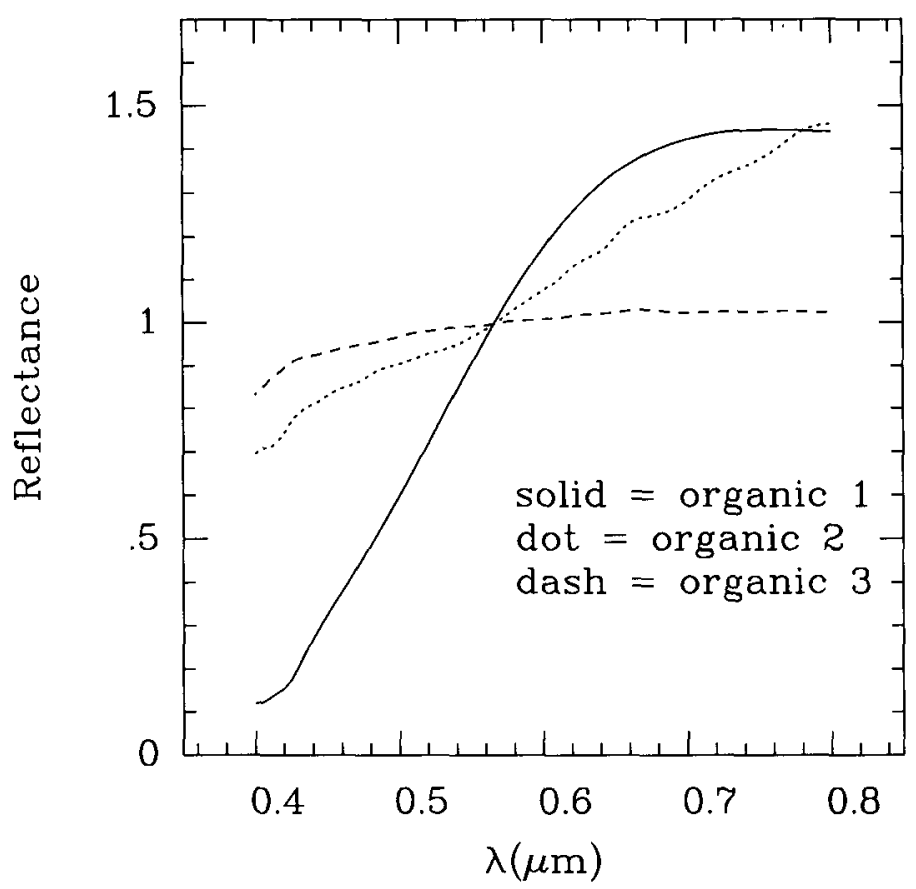

Figure 5. The reflectance spectra $(0.4$ to $0.8 \mu \mathrm{m})$ of three laboratory synthesized organic samples are shown. The spectra were obtained at room temperature and were normalized to 1 at $0.56 \mu \mathrm{m}$. Organics 1,2 and 3 were produced by bombarding benzene ice $(77 \mathrm{~K})$ with $10^{15}$ ions $/ \mathrm{cm}^{2}\left(100-\mathrm{keV} \mathrm{He}^{+}\right)$, benzene ice $(77 \mathrm{~K})$ with $10^{15}$ ions $/ \mathrm{cm}^{2}(200-\mathrm{keV}$ $\left.\mathrm{Ar}^{+}\right)$, and methane ice $(10 \mathrm{~K})$ with $10^{17} \mathrm{ions} / \mathrm{cm}^{2}(1.5-\mathrm{MeV} \mathrm{H})$, respectively.

A summary of the evolution of frozen methane under bombardment by $\mathrm{MeV}$ protons is given in Table 3.

\section{Theory}

The incident ions lose their energy to the materials of interest in part by colliding with atoms in the material and by inducing nuclear reactions. However, the predominant energy loss over a broad range of ion energies is to electronic excitations and ionizations. All of these forms of energy lead eventually to both bond breaking and molecular motion. If atoms or molecules are set in motion in the surface layer, they may leave the solid (sputter) (Brown and Johnson 1986), whereas if this happens below the surface, it contributes to ion-induced diffusion and/or production of voids. The bond breaking and subsequent formation of new bonds lead to the formation of new species in the solid, as discussed, accounting for ion-induced chemical alteration of solids. 


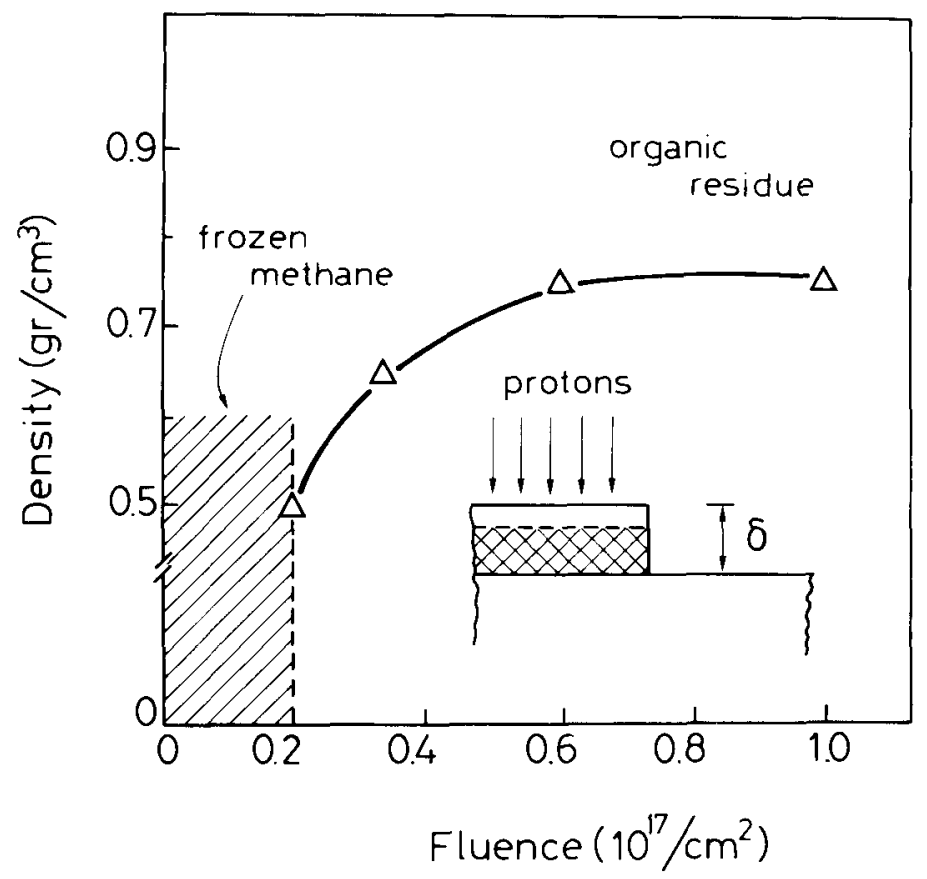

Figure 6. The density of a solid organic residue obtained by bombarding $\mathrm{CH}_{4}$ ice with $1.5-\mathrm{MeV}$ of $\mathrm{H}^{+}$is plotted versus the proton fluence.

The electronic processes that lead to the material alteration can also be induced by energetic electrons and energetic photons. Incident electrons behave similarly to fast protons except that they are more readily deflected because of their small mass and, hence, do not penetrate into the material as efficiently. The often dominant UV and EUV photons differ from the fast ions in that they produce single excitation/ionization events and the average penetration depths $\left(10^{17}\right.$ to $\left.10^{19} \mathrm{~mol} / \mathrm{cm}^{2}\right)$ are larger than solar wind ions, but much less than that of a MeV proton $\left(10^{20} \mathrm{~mol} / \mathrm{cm}^{2}\right)$.

The rate of energy loss by fast ions in a solid is given in terms of the stopping power of the material, $\mathrm{dE} / \mathrm{dx}$, the energy loss per unit path length. In Figure 8 (dashed line), this is given for the dominant electronic energy loss by protons in $\mathrm{H}_{2} \mathrm{O}$ scaled to the mass density of the solid. At energies $\simeq 1 \mathrm{GeV}$, of interest for cosmic rays, the mean-freepath for inelastic nuclear interactions is $\simeq 100 \mathrm{~g} / \mathrm{cm}^{2}$ (i.e., it is 1 meter at a density of $1 \mathrm{~g} / \mathrm{cm}^{3}$ ). Therefore, near the minimum of the electronic energy loss function, the nuclear reactions will also contribute (e.g., Ryan and Draganic 1987). For other solids, the electronic and nuclear reaction contributions scale roughly as the mass density, which is nearly proportional to the number of electrons and nucleons. (Note: As the mass scaling is poor for $\mathrm{OH}$-containing species, the results for other species (e.g., $\mathrm{CO}_{2}$ and $\mathrm{SiO}_{2}$ ) should be 


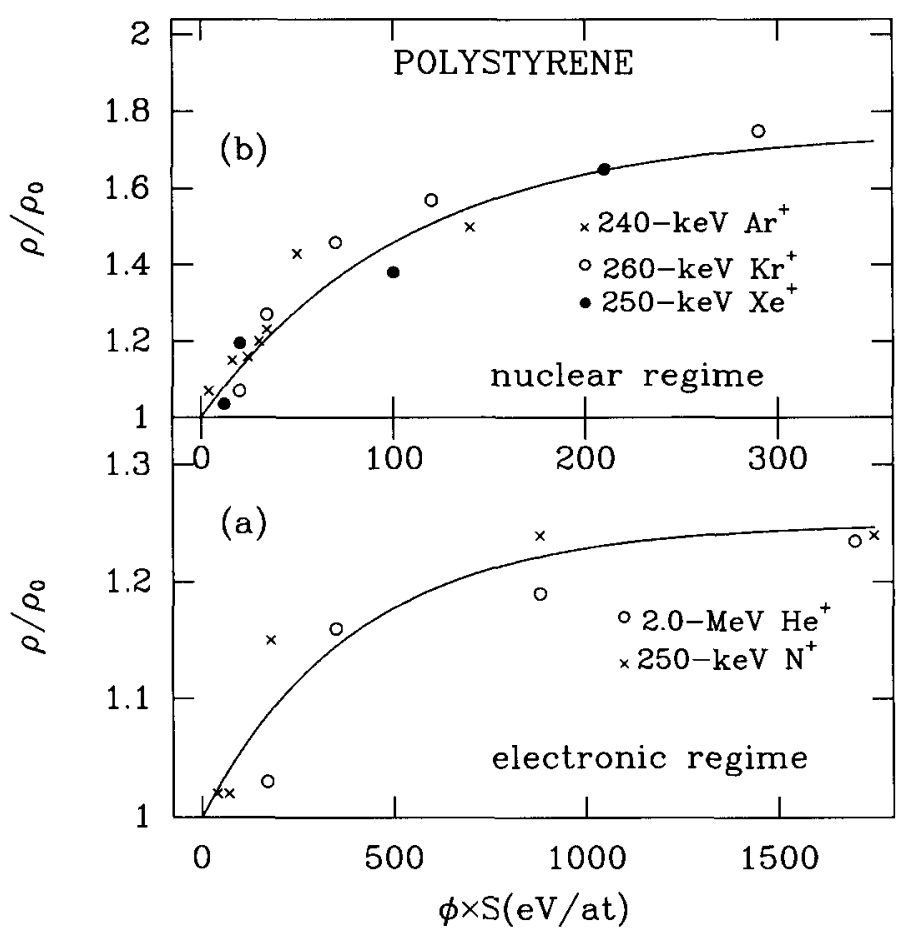

Figure 7. The density variation of polystyrene by ion irradiation is shown versus the deposited energy (eV/at) for the two regimes of energy deposition: (a) electronic and (b) nuclear.

divided by 1.3 after scaling by mass.) The rough penetration depths are also given in Figure 8 (full line).

As the effects described (sputtering and chemical change) all depend on the energy deposited, laboratory measurements can be usefully scaled using $\mathrm{dE} / \mathrm{dx}$ (or the stopping cross section $\left.S=\rho^{-1}(\mathrm{dE} / \mathrm{dx})\right)$. For example, the laboratory sputtering yields for low-temperature $\mathrm{H}_{2} \mathrm{O}$ and $\mathrm{CO}$ are $\sim 3 \times 10^{-3} \mathrm{~S}_{\mathrm{e}}^{2}$ and $4 \times 10^{-2} \mathrm{~S}_{\mathrm{e}}^{2}$ molecules per ion, respectively, for ions with energies $>1 \mathrm{keV}$, where $S_{\mathrm{e}}$ is the electronic part of the stopping cross-sections. Therefore, the more volatile species is sputtered more readily, but the most energetic ions (small $S_{e}$ ) do not sputter either material very efficiently. On the other hand, at low excitation densities, the material changes are proportional to $S_{\mathrm{e}}$ (viz., Figure 3 ). Therefore, for the fastest ions, material alterations dominate (although there may be a small linear component to the yield also; see, e.g., Brown and Johnson 1986). In making these alterations, however, sputtering always plays a critical role. That is, in a closed system, the material would eventually reach a new stable equilibrium mix of the original material and new species. Only due to the presence of the vacuum interface, through which species are permanently lost, will the material be driven continuously to a "final" state. 
Table 3. Summary of the evolution of frozen methane under bombardment by MeV protons at $10 \mathrm{~K}$.

\begin{tabular}{cllll}
$\begin{array}{c}\text { Dose } \\
\mathrm{H}^{+} / \mathrm{cm}^{2}(\mathrm{eV} / \mathrm{mol})\end{array}$ & \multicolumn{1}{c}{ / $/ \mathrm{H}^{(\mathrm{a})}$} & Resulting Material(b) & Color & Density $(\mathrm{c})$ \\
\hline $0(0)$ & $1 / 4$ & Frozen methane $(\sim 10 \mathrm{~K})$ & Clear & 0.5 \\
$2 \times 10^{16(100)}$ & $1 / 3(1 / 1)$ & Org. residue $50 \%(\sim 300 \mathrm{~K})$ & Yellow & 0.5 \\
$5 \times 10^{16(250)}$ & $1 / 2(1 / 0.7)$ & Org. residue $75 \%(\sim 300 \mathrm{~K})$ & Brown & 0.5 \\
$1 \times 10^{17}(500)$ & $1 / 1.5(1 / 0.5)$ & Carbonaceous $90 \%(\sim 300 \mathrm{~K})$ & Black & 0.75
\end{tabular}

(a) The $\mathrm{C} / \mathrm{H}$ ratios resulting after heating to $\sim 300 \mathrm{~K}$ are shown in parentheses.

(b) The percents shown are approximate fractions of the original carbon in the residue after warming.

(c) This is the density of a fluffy aggregate; the density of a single subaggregate presumably may be much higher.

It is important to note that the alterations are induced by discrete energy deposition events at low $(\mathrm{dE} / \mathrm{dx})_{\mathrm{e}}$. Typically, the number of such events (generally ionizations) per unit path length for fast ions and electrons is $(\mathrm{dE} / \mathrm{dx})_{\mathrm{e}} / \mathrm{W}_{\mathrm{e}}$, where $\mathrm{W}_{\mathrm{e}} \sim 30 \mathrm{eV}$ (e.g., Johnson $1989 \mathrm{a}, 1989 \mathrm{~b})$. Therefore, low (dE/dx) implies few events per unit path length, each of which can contribute to change. For fast ions and electrons, these occur, on the average, in pairs: an ionization (the average energy expended is 30 to $60 \mathrm{eV}$ ) plus a fast electron leading another ionization. In addition, it is well-known that the ions always deposit a high-energy density at the end of their range (a Bragg peak). The high density of multiple bond breaking in the very short time scale means that a small fraction of the energy of even the fastest ions can lead to severe local alterations (Strazzulla 1986). Of course, nuclear energy-loss events, which generally result in a number of $\mathrm{MeV}$ particles, produce a high ionization density randomly along the track of a GeV ion. For these reasons, the results for $\mathrm{MeV}$ ions can be extrapolated to results for much faster ions.

A key element in the above is the storage time for damage. Reimann et al. (1984) and others have shown that low-temperature ices "remember" their radiation history. That is, on bombarding an ice sample, the yield of $\mathrm{O}_{2}$ is found initially to be fluence-dependent. That is, the first ions through alter the sample, and the next complete the reactions and eject the new species. Turning the beam off for times on the order of minutes to hours (the times are limited by the quality of the vacuum) the fluence dependence does not return, i.e., $\mathrm{O}_{2}$ is promptly emitted at the successive beam-on. Therefore, the precursor species to $\mathrm{O}_{2}$ ejection from water ice remain stored in the ice. In addition, electron-spin-resonance studies of radical species in ices irradiated at very low temperatures show that these radicals 


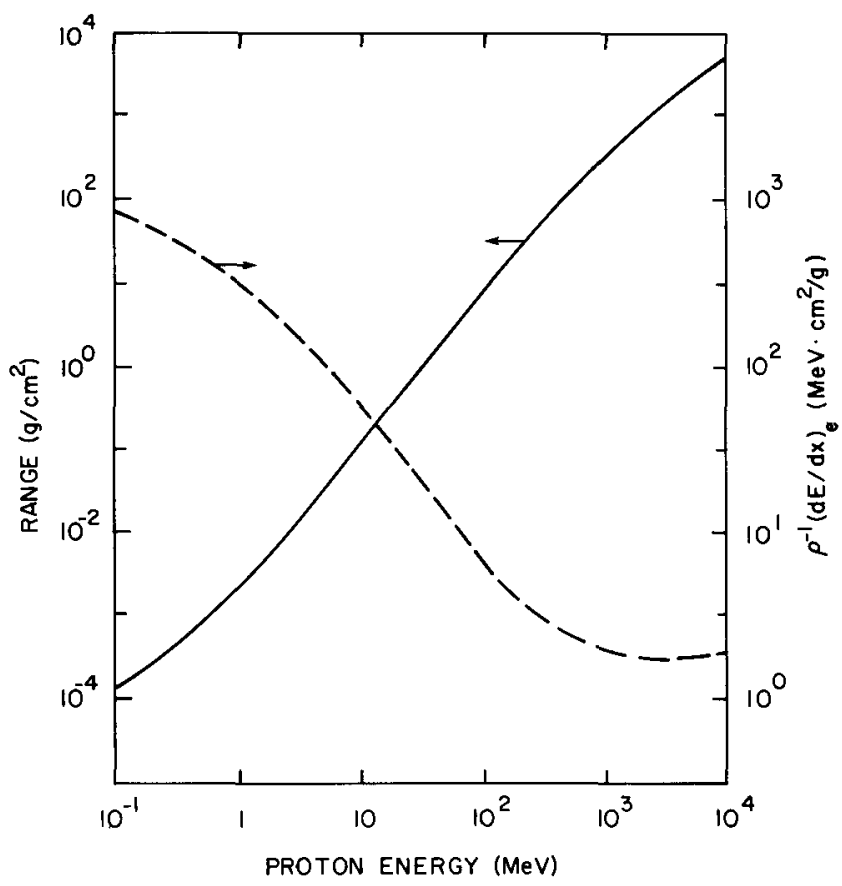

Figure 8. The dashed curve is the electronic energy loss per unit path length of a fast ion in water. The solid curve is the ion penetration depth obtained using the solid curve (no nuclear interactions).

rapidly disappear as temperature is increased above $100 \mathrm{~K}$ (Hart and Platzman 1961). Since extrapolation of such results to astrophysical time scales is dangerous, more detailed laboratory studies are needed. It is generally agreed upon that most radical species are "very" stable at temperatures of about $10 \mathrm{~K}$, so that in the interstellar medium or the Oort cloud, the fluence-dependent changes observed in the laboratory should occur for incident photons, electrons, and ions. Therefore, to first order in such regions, the various radiations can be compared according to their net energy deposited. On the other hand, the ions may produce unique alterations in the high-excitation density volumes discussed above and at higher temperatures (e.g., the inner Solar System), where radical storage is more problematic.

Because of the problem of storage times discussed above, experiments must be carried out at an irradiation flux low enough to avoid any heating of the sample. Enhanced material alterations due to beam-induced heating are well-established (e.g., Mazzoldi and Arnold 1987). Since the sample is always connected thermally to a heat sink, even in the insulating samples studied, conduction to the substratum usually dominates radiative loss. For these reasons, it is clear that the results of discharge experiments and experiments using a high flux of UV may have artifacts that limit the applicability to the processes of 
interest in comets. For these reasons, there is a strong need for quantitative results under well-characterized conditions in the next generation of experiments. Sack et al. (1988) have clearly shown the beam current effect.

In a recent set of experiments on methane (Brown et. al. 1987), the origin of the structural changes produced in ices at low ion-fluence was made clear. On monitoring the $\mathrm{H}_{2}$ ejected from methane irradiated by MeV protons, a "threshold" fluence was found, after which the $\mathrm{H}_{2}$ loss increased dramatically. This was interpreted to be due to a percolation effect. That is, events produced by the incident ions throughout the penetration depth of the solid produced defects (voids) in the material and also produced $\mathrm{H}_{2}$ trapped in these defects. On further irradiation, connecting paths were eventually formed, allowing the loss of volatile species down to the depth of the ion penetration. By this means, the material is everywhere connected to the vacuum. This interpretation was confirmed recently in a series of experiments on the changes of reflectance of the initially transparent deposits, in which the results are best interpreted by the production of voids in both water and methane ice. The accumulation of these was also seen in an electron microscope (Johnson et al. 1985).

The above process, therefore, produces a surface that is light-scattering, fluffy, and under-dense (Strazzulla et al. 1988). If such a material contains a hydrocarbon, a "lattice" of long-chain molecules is also formed, so that, on warming, a fluffy residue is obtained (Foti et al. 1984). As the volatile material is continuously driven from the solid, the material remains with large voids (fluffy). However, locally, the density increases, becoming more graphitic or even diamond-like (densities of $4 \mathrm{~g} / \mathrm{cm}^{3}$ locally), increasing the average density.

\section{Applications}

In the last 10 years, there have been many applications of the above experimental results, not only for comets and cometary debris, but also for interstellar and protostellar grains, and solid bodies in the outer Solar System. Reviews of these latter arguments can be found in recent literature (Strazzulla 1988, Johnson 1989a). debate are:

Among the cometary applications that have been discussed and/or are objects of

(1) The characteristics of ion-synthesized organic material and the comparisons with astrophysical observations and other formation mechanisms have been extensively debated. After the findings of the recent Halley's passage, this is particularly important, as discussed in Section 7.

(2) It has been suggested (Lanzerotti et al. 1978, Johnson et al. 1983, Strazzulla et al. 1983b) that beyond 5 to $6 \mathrm{AU}$, fast ions from the solar wind become the most important agents of erosion. This is shown in Figure 9, where the number of molecules released from an $\mathrm{H}_{2} \mathrm{O}$-dominated object and a $\mathrm{CO}_{2}$-dominated object are given (versus the solar distance) as being due to sublimation and ion sputtering for ions at average solar activity (e.g., Mukai and Schwehm 1981). The results in Figure 9 show that ion sputtering becomes the major erosion mechanism at distances greater than 5 to $6 \mathrm{AU}$. For $\mathrm{CO}_{2}$, there is also an enhanced release of material due to prior irradiation. The net yields, however, are low, as are the penetration 


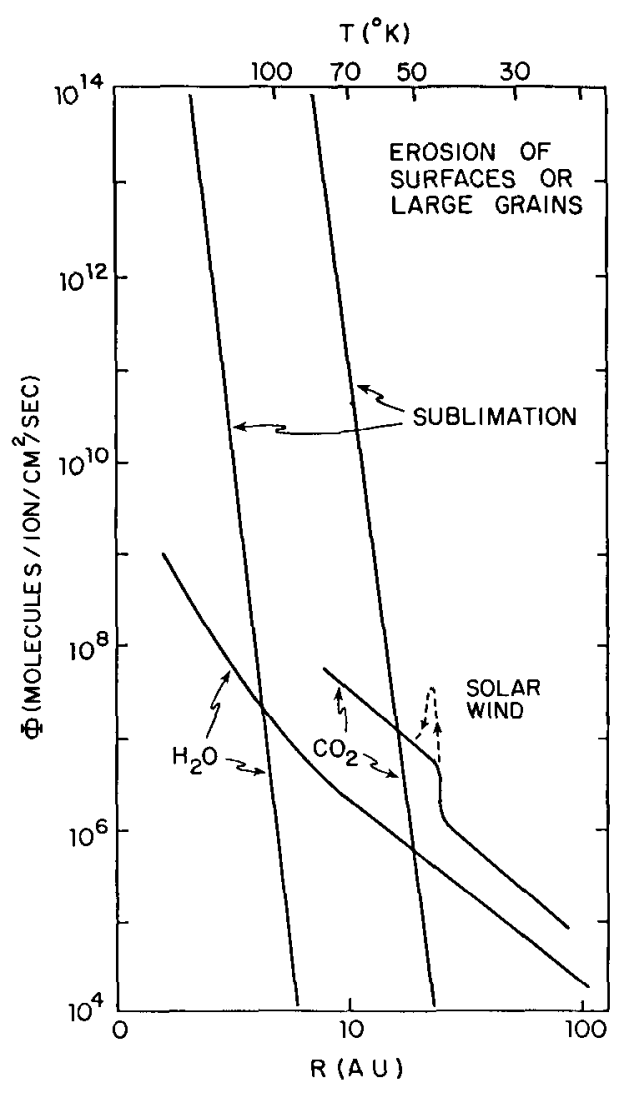

Figure 9. Comparison of the flux of molecules from the surface of an icy (water or carbon dioxide) object due to sublimation and solar wind sputtering. The dashed curve indicates enhanced activity due to any accumulated irradiation of $\mathrm{CO}_{2}$ (from Brown et al. 1982a).

depths, and therefore they primarily affect cometary debris, as discussed in Section 9. However, in a T-Tau phase, these yields become important, as described in Section 7.

(3) The possibility that specific molecules such as $\mathrm{H}_{2} \mathrm{CO}$ (Pirronello et al. 1982, Pirronello et al. 1984) or $\mathrm{N}_{2} \mathrm{H}_{4}$ (Strazzulla 1985) are created by radiation, then stored for a long time and finally released during the approach of a comet near the Sun has been discussed. Interestingly, $\mathrm{H}_{2} \mathrm{CO}$ has been detected, e.g., in comet IRAS-Araki-Alcock (Cosmovici and Ortolani 1984) and, tentatively, in comet Halley (Moroz et al. 1987), as have possible polymers of $\mathrm{H}_{2} \mathrm{CO}$ (Huebner 1987). $\mathrm{N}_{2} \mathrm{H}_{4}$ has been invoked many times as the parent molecule of $\mathrm{N}_{2}^{+}$and $\mathrm{NH}_{2}$, commonly observed in cometary comas (e.g., Yamamoto et al. 1983). It has been suggested recently that through the measurement of the abundance of $\mathrm{H}_{2} \mathrm{CO}$ in the 
nuclei of long period $\left(\simeq 10^{6} \mathrm{yr}\right)$ comets, it could be possible to deduce information on the low-energy component of cosmic radiation that cannot be measured from inside the heliosphere (Pirronello and Lanzafame 1989). The upper several meters of comets exposed to radiation in the Oort cloud are altered (Donn 1976, Whipple 1977). This region is enriched in longchain stable organic material and radical species. Johnson at al. (1987) argued that a refractory crust can be formed, which may become a permanent feature of a comet injected into the inner Solar System. When a new comet enters the inner Solar System, there will be early activity, initial fissures in the organic crust, and the break-off of unstable pieces of the crust, due to warming of subsurface volatiles and radicals. If this comet enters a periodic orbit in the inner Solar System, the remaining mantle should be continuously hardened due to thermal processing and accreting of dust from active regions (Prialnik and Bar-Nun 1988). There will also be permanently active regions that were initially shaded from the cosmic ray radiation when the comet was in the Oort cloud or that subsequently lost their crust. The structure of the surface with depth in the Oort cloud is discussed in Section 7.

\section{Effect Versus Time, Effect Versus Depth}

In Figure 10, we present the lifetimes (during the T-Tau phase) of pure ice grains as a function of grain radius, at two distances from the Sun (Strazzulla 1985). The solid lines refer to sublimation (the luminosity of the young Sun was assumed to be $20 \mathrm{~L}_{\odot}$ ), and the dashed lines to ion erosion. As in Figure 9, "sputtering" is the main mechanism of destruction at larger distances. Here, however, the results for grains larger than $2 \mu \mathrm{m}$ dominate at $9 \mathrm{AU}$ and larger than $50 \mu \mathrm{m}$ at $4.5 \mathrm{AU}$. In fact, at distances greater than $9 \mathrm{AU}$, erosion by particles is the only effective mechanism of destruction in the time period of interest. Moreover, during the $10^{6} \mathrm{yr}$ assumed for the duration of the T-Tau phase, grains as large as $1 \mathrm{~cm}$ (at $4.5 \mathrm{AU}$ ) and $0.2 \mathrm{~cm}$ (at $9 \mathrm{AU}$ ) can be completely destroyed and the released material removed by the interaction with the intense $\mathrm{T}$-Tau wind $(\mathrm{v} \sim 100 \mathrm{~km} / \mathrm{s})$. Because, in the $10^{6} \mathrm{yr}$, the wind by itself only blows out those particles with a radius $<10^{-2} \mathrm{~cm}$ (Harris 1978), the sputter removal is important for destroying grains two orders of magnitudes larger.

As it is very likely that grains different from pure ice exist, chemical alterations (volume processes along the entire ion track) may be more important than surface or chemical sputtering. Let us consider, as an example, the fate of a grain consisting of a silicate core covered with $\sim 40 \mu \mathrm{m}$ of frost containing, say, $40 \%$ of a hydrocarbon (e.g., Greenberg 1982) and bombarded by the flux of ions assumed above. The chosen thickness corresponds to about the range of $1.5-\mathrm{MeV}$ protons in a molecular solid containing $\mathrm{C}$, $\mathrm{N}$, and $\mathrm{O}$ with a density of $\sim 1 \mathrm{~g} / \mathrm{cm}^{3}$.

In Figure 11, the assumed flux of protons $(E>1 \mathrm{MeV})$ and the time $t$ required to polymerize $90 \%$ of the carbon atoms according to Eq. (2) are shown versus the solar distance. It is seen that $90 \%$ of the carbon atoms are bound together in time scales smaller than the $10^{6} \mathrm{yr}$ assumed for the duration of the T-Tau phase, for distance as large as a few times $10^{3} \mathrm{AU}$. In addition, for particle flux two orders of magnitude lower, the process 


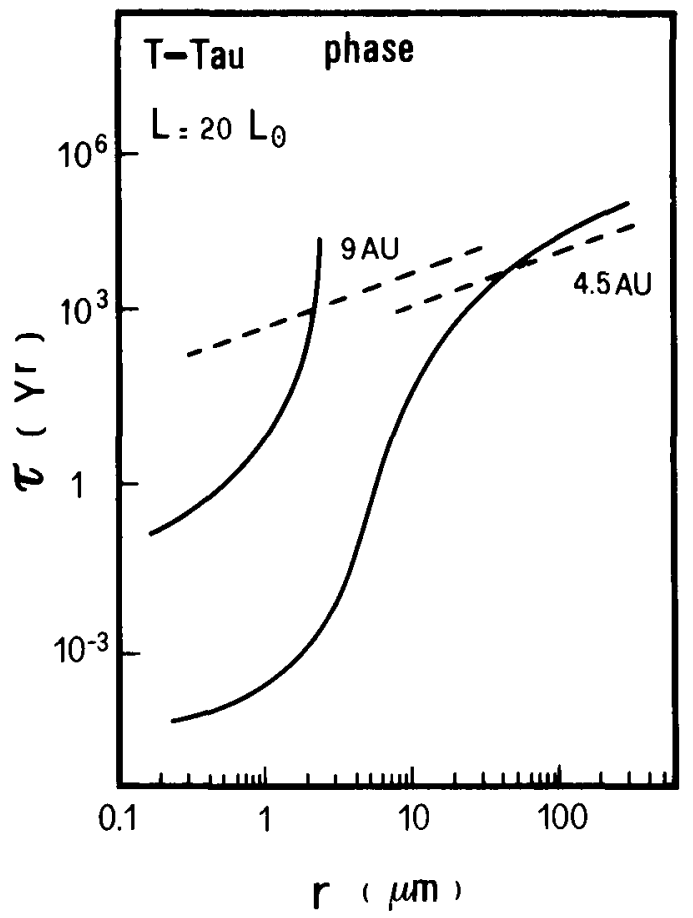

Figure 10. Lifetimes, at two different distances from the Sun during the T-Tau phase, of ice grains versus grain radius: against sublimation $(-)$ and $\mathrm{MeV}$ ion bombardment ( - - ) .

works up to distances $>10^{2} \mathrm{AU}$ (Strazzulla 1985), i.e., well beyond Pluto and the probable region (d $\sim 50 \mathrm{AU}$ ) of comet formation (see, e.g., Whipple 1972).

Using the data discussed in Section 5 , the energy deposited by a given ion spectrum versus the depth in a comet can be calculated. Thus, it is possible to evaluate the grade of alteration, exemplified by the polymerized fraction of carbon, of cometary material versus depth.

Figure 12 gives approximate dose versus depth curves for cosmic ray irradiation of the mantle of a comet in the Oort cloud. The solid upper curve is obtained using the cosmic ray ionization rate measured in the atmosphere (see Whipple 1977, Johnson 1989b). The upper solid curve is the calculation of Ryan and Draganic (1987) for E > $1 \mathrm{GeV}$, combined with the results of Moore (1982) for $\mathrm{E}<1 \mathrm{GeV}$. The latter results provide lower limits to the dose at small depths, and the results obtained from air provide lower limits at large depths. Thus, in Figure 12, the dashed line interpolates between these (see Johnson 1989b). Assuming that $\simeq 30 \mathrm{eV}$ is deposited per ionization, the right-hand axis gives the number of times, on the average, an original small molecule has been ionized. In Figure 12, the depths are given in $\mathrm{g} / \mathrm{cm}^{2}$. The density of Halley's comet has been evaluated to be between 0.008 and $0.24 \mathrm{~g} / \mathrm{cm}^{3}$ by Wood (1986) and Rickman (1986) and 


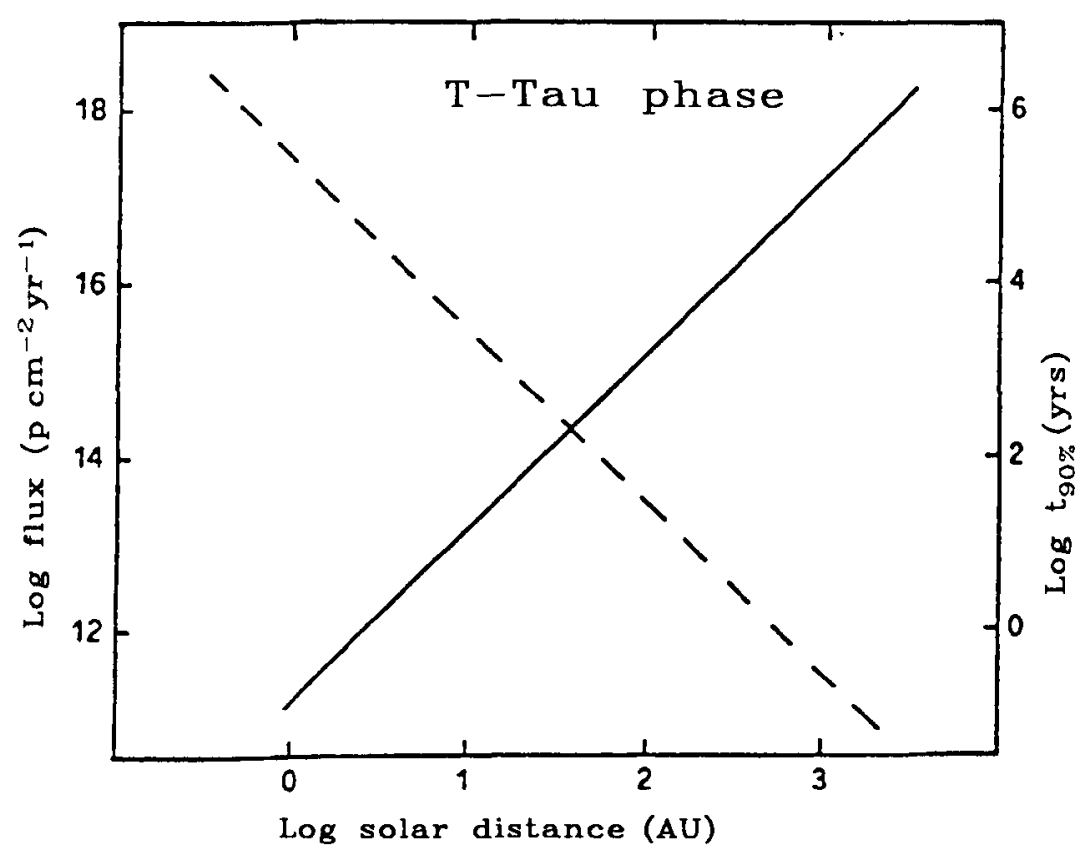

Figure 11. The proton $(\mathrm{E}>1 \mathrm{MeV})$ flux $(--)^{-}$and corresponding time ( - ) required to polymerize $90 \%$ of the carbon atoms contained in the "ideal" grain considered in the text, versus solar distance.

$\simeq 0.6 \mathrm{~g} / \mathrm{cm}^{3}$ by Sagdaeev et al. (1988). In Figures 12 and 13 , we use $0.2 \mathrm{~g} / \mathrm{cm}^{3}$. In Figure 13, the solid curve is the fraction of cross-linked carbon versus depth. The fraction is obtained from Eq. 2, with $\sigma \phi=\mathrm{D}_{\mathrm{M}} /(140 \mathrm{eV}$ ) (or, equivalently, $\mathrm{G} \simeq 0.7$ ). Water molecules are depleted from the mantle because of sputtering and chemical alteration $\left(\mathrm{H}_{2} \mathrm{O} \rightarrow \mathrm{H}_{2}+1 / 2 \mathrm{O}_{2}\right)$. For this process, using $\alpha$-particles, one gets $\sigma \phi=$ $\mathrm{D}_{\mathrm{M}} /(140 \mathrm{eV})$ (or $\left.\mathrm{G} \simeq 0.7\right)$, and using $\beta$-particles, $\sigma \phi=\mathrm{D}_{\mathrm{M}} /(300 \mathrm{eV})($ or $\mathrm{G} \simeq 0.3)$. For the fast protons, we use the latter as a lower limit that is plotted versus depth in Figure 13 (dashed curve). From Figure 13, it is clear that for a noteworthy thickness of cometary material, a large fraction of $\mathrm{C}$-atoms are linked together to form a refractory material. This fraction is between $\simeq 30 \%$ (at $5 \mathrm{~m}$ ) and $\simeq 10 \%$ (at $20 \mathrm{~m}$ ) for $\rho=$ $0.2 \mathrm{~g} / \mathrm{cm}^{3}$. In addition, Al radioactivity gives a contribution, on the order of few percent throughout the entire comet, to the amount of linked carbon formed by earlier processing.

The irradiation dose received in the outer $10 \mathrm{~g} / \mathrm{cm}^{2}$ is sufficient to make a stable, cohesive crust with a significant tensile strength. Below this, down to about $100 \mathrm{~g} / \mathrm{cm}^{2}$, is a material that is depleted in volatiles, but that has a considerable organic fraction and has unrecombined radicals, which can react on heating. At greater depths, down to about $300 \mathrm{~g} / \mathrm{cm}^{2}$, volatiles remain, but the original ices have experienced significant bond breaking and chemical alteration. This bond breaking and reformation (equivalent to heating) partially destroys the memory of the formation region. Recently, Mumma et al. (1989) 


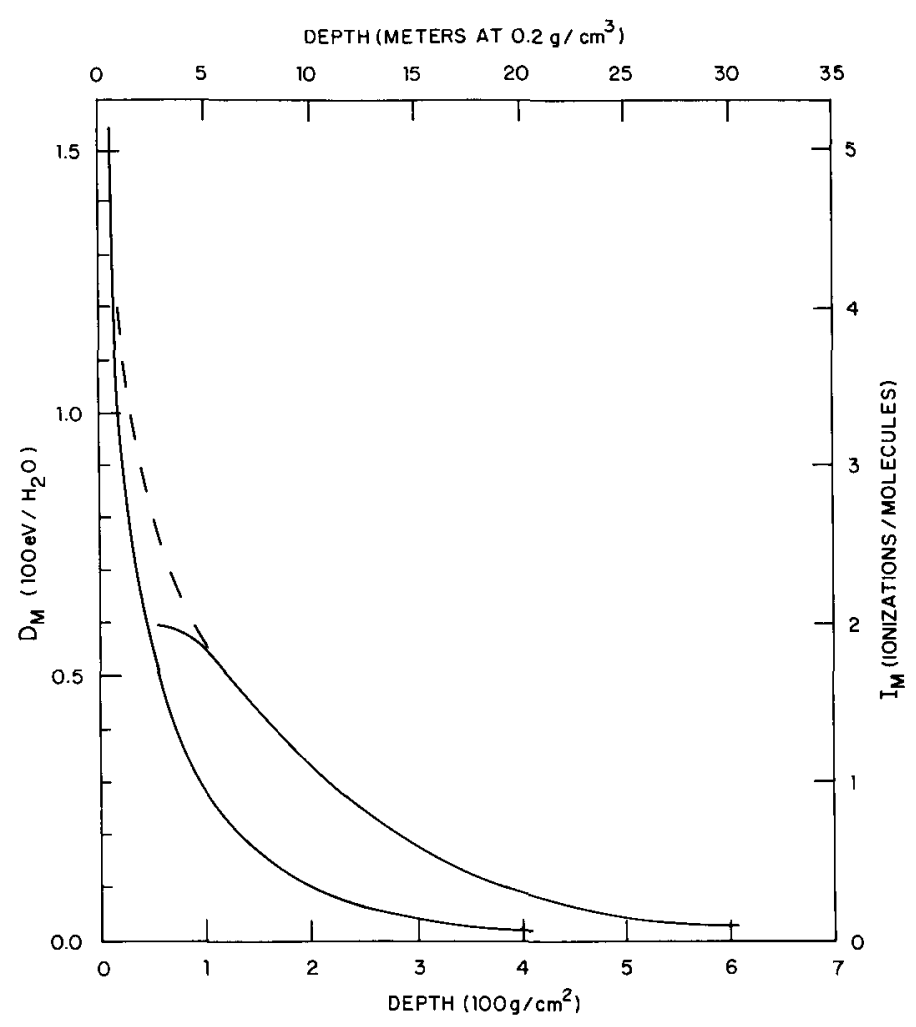

Figure 12. Dose versus depth for a comet in the Oort cloud. The solid lower line represents data based on atmospheric measurements, and the solid upper line is from Ryan and Draganic (1987) and Moore (1981). The dashed line is based on interpolation (see Johnson 1989b).

pointed out that an ortho-para $\mathrm{H}_{2} \mathrm{O}$ ratio of about 2.3 in the $\mathrm{H}_{2} \mathrm{O}$ from Halley is indicative of a formation temperature of about $25 \mathrm{~K}$. In contrast, for comet Wilson, the authors found a ratio of $\simeq 3.2$, consistent with the statistical ratio 3 (Mumma et al. 1989) that would occur at high temperatures. As Wilson is a new comet, they attributed this difference to cosmic-ray alteration of the outer icy layers. That is, dissociative ejection of $\mathbf{H}$ from $\mathrm{H}_{2} \mathrm{O}$, followed by pickup of another $\mathrm{H}$ to reform $\mathrm{H}_{2} \mathrm{O}$, will destroy the ortho-para ratio. The estimates above make this explanation plausible, although, of course, other surface "heating" processes in the Oort cloud might also produce such an effect (e.g., Stern and Shull 1988, Stern 1988). Based on the above, a layer on the order of at least $500 \mathrm{~g} / \mathrm{cm}^{2}$ is so altered. 


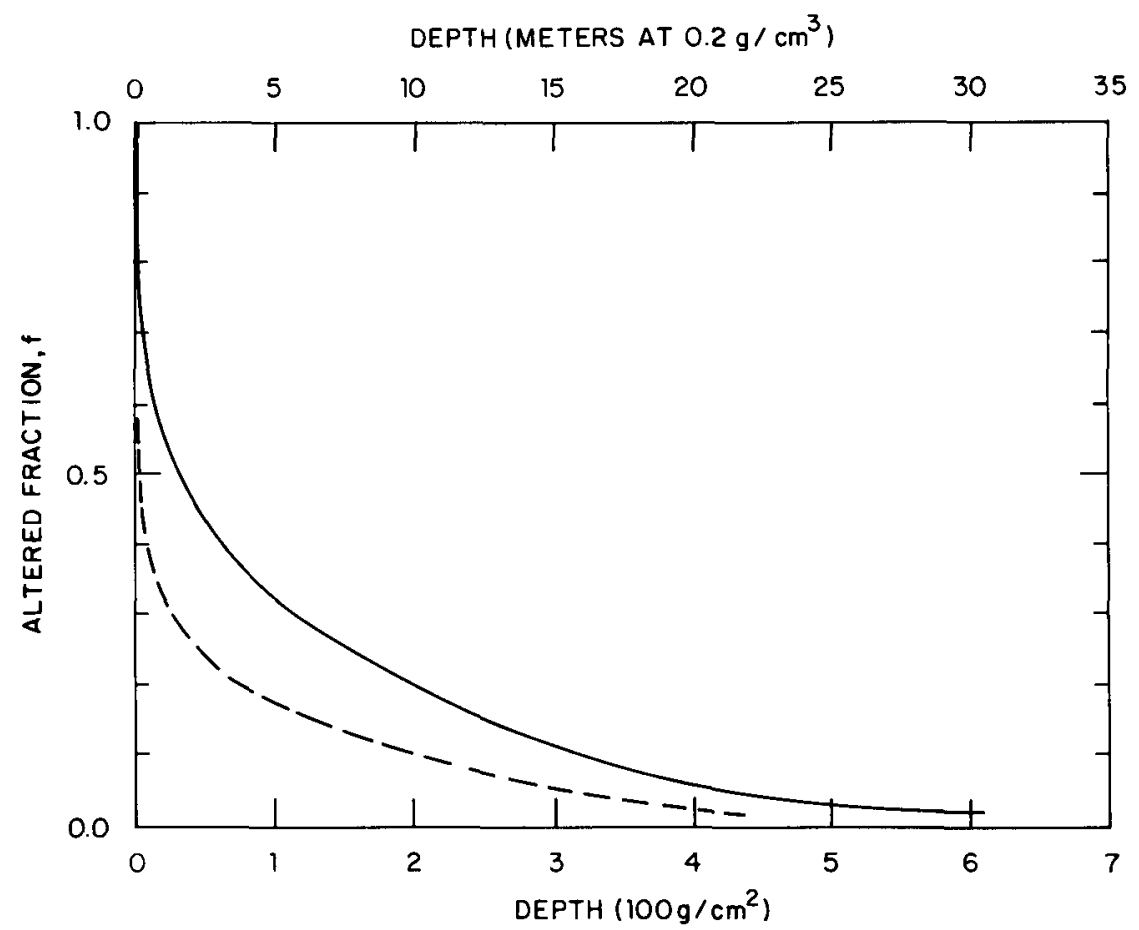

Figure 13. The polymerized fraction (in $4.6 \times 10^{9} \mathrm{yr}$ ) of carbon versus the depth into a comet and the lower limit to the fraction of water lost by chemical alteration.

\section{Organics in Comets}

\subsection{OBSERVATIONS}

Before Halley's recent passage, evidence was reported already for the presence, in comet Crommelin, of dark grains possibly related to organic radiation-processed ices (Hanner et al. 1985). However, spacecraft and ground-based observations of comet Halley provided overwhelming evidence of the presence of a large fraction of about $30 \%$ (in mass) of organic material.

PUMA and PIA experiments on board VEGA and Giotto gave evidence for the presence of complex radiation-processed dust particles (Kissel et al. 1986a, 1986b). Those organic materials are probably present even as refractory mantles around silicate cores (Kissel and Krueger 1987a, Jessberger et al. 1988).

From the PICCA experiment, evidence for chain molecules enriched in carbon, hydrogen, and oxygen was found (Mitchell et al. 1987). Huebner (1987) interpreted those data as evidence for the first polymer (short polymer chains of paraformaldehyde) in space.

Infrared emission bands, typical of organic materials, were observed at 3.3, 3.4, and $7.5 \mu \mathrm{m}$ from the IKS-VEGA experiment (Combes et al. 1986, Moroz et al. 1987). 
Emission features around 3.2 to $3.6 \mu \mathrm{m}$ were also reported, from ground-based observations, by several groups (Wickramasinghe and Allen 1986, Baas et al. 1986, Knacke et al. 1987, Tokunaga et al. 1987). Airborne and ground-based spectrophotometry in the 5- to $13-\mu \mathrm{m}$ range showed, behind the strong, broad silicate emission band at $10 \mu \mathrm{m}$, a weak feature at $6.8 \mu \mathrm{m}$ attributed either to carbonates or to $\mathrm{C}-\mathrm{H}$ deformation modes in organics (Bregman et al. 1987).

It is interesting that, after Halley's passage, organic grains were also discovered in comet Wilson (a new comet!) via the 3.4- $\mu \mathrm{m}$ emission feature (Allen and Wickramasinghe 1987). It is important to note that, based on purely spectroscopic grounds, it is not possible to distinguish between different molecules responsible for this emission, nor is it possible to say if the molecules emitting the 3.4- $\mu \mathrm{m}$ feature are free (gas phase) or embedded in solid matter. However, the broad appearance of those bands and the findings from the PUMA, PIA, and PICCA experiments favor the hypothesis that at least a large fraction of those emissions are solid-state features.

\subsection{MODELS}

The presence in comets of the following different kinds of organics and different formation processes has been suggested in recent literature:

(1) Biological grains (e.g., Hoyle and Wickramasinghe 1985).

(2) Organic residues from electrical discharges of, e.g., methane ice clathrate (e.g., Chyba and Sagan 1987).

(3) UV-irradiated pre-cometary grains (e.g., Greenberg 1982).

(4) Residues from ion-irradiated ices (e.g., Strazzulla 1986).

An acceptable process for the formation of cometary organics not only has to reproduce (or at least not contradict) the observations, but also has to explain the formation and evolution of organics in comets based on laboratory results. Of course, there is the possibility that more than one formation process contributes to the total organic budget.

Hoyle and Wickramasinghe (1987) claimed that the observed $3.4-\mu \mathrm{m}$ band (Wickramasinghe and Allen 1986) is fitted by bacteria heated to $320 \mathrm{~K}$. Many groups have strongly criticized those conclusions, and we concur. Kissel and Krueger (1987b) did not find, from PUMA data, any phosphorus and only $10^{-6}$ sodium and potassium in the organics. This is in contrast with the fact that freeze-dried cells should contain at least a percent of sodium and $0.1 \%$ of potassium. Chyba and Sagan (1988) and Greenberg and Zhao (1988) criticized the way Hoyle and Wickramasinghe obtained a fit of the 3.4- $\mu \mathrm{m}$ band. In fact, Halley's spectrum requires the choice of different temperatures for the continuum $(\simeq 320 \mathrm{~K}$ at $1 \mathrm{AU})$ and for the $3.4-\mu \mathrm{m}$ band caused by particles much hotter than the continuum $(\sim 500 \mathrm{~K}$ at $1 \mathrm{AU})$. Moreover, it is far from clear how bacteria can be formed, and, if formed, how they can be stored in a comet, considering that radiation is extremely detrimental to their survival.

The importance of discharges is based on the observation of the formation of organics in "discharge" experiments and on the more or less implicit assumption that discharges may produce effects similar to those of single incoming energetic ions (Khare et al. 1984). Such an assumption does not seem to be fully justified and requires more accurate laboratory checks. In any case, either discharge experiments are not equivalent to ion irradiation 
experiments or process (2) is equivalent to (4). In the former case, there is little evidence for the importance of discharges for any cometary materials. In the latter case, ion beam experiments have a simplicity in their interpretation that allows one to obtain quantitative results useful for interpreting cometary data.

The processes for organic material formation-processes (3) and (4)_are similar, as discussed earlier. Both UV (e.g., Greenberg 1982) and ions (Strazzulla et al. 1983a) can build up organic mantles on interstellar grains. Assuming that the ion fluxes are those given in Section 1, UV irradiation is predominant in the interstellar medium. In fact, much of the UV irradiation is produced by cosmic-ray energy loss in the gas. Thus, if comets are a direct aggregate of interstellar dust, they may be rich in UV-processed materials. In this case, ions alter the existing organics and ices during the T-Tau phase and form a severalmeter-deep organic crust in the Oort cloud. On the other hand, if comets are not an aggregate of interstellar dust, then ions become the only serious candidate for forming complex radiation-processed organics. Greenberg and co-workers have given arguments favoring the hypothesis of a direct aggregation from interstellar dust into comets. However, this question is far from resolved.

\section{Cometary Debris}

It is widely thought that interplanetary grains are predominantly cometary debris. After the spacecraft missions (e.g., Kissel and Krueger 1987a), it has also been presumed that most of the cometary grains are a fluffy agglomerate of silicate core-organic mantle grains. After leaving the parent comet, they are modified by heating, UV photodesorption (Combi 1987), and solar ion irradiation. The first two processes drive off the most volatile species, generally soon after ejection from the nucleus. Subsequently, during the time the grains spend $\left(10^{4}\right.$ to $\left.10^{5} \mathrm{yr}\right)$ before entering the upper atmosphere of the Earth or colliding with the Moon's surface, they are effectively modified by solar ions. One effect of sputtering in the interplanetary medium is the driving off of residual volatiles. This is done efficiently by the solar wind and solar flare ions, due to their full penetration depth (Johnson and Lanzerotti 1986). The sputtering yields of organics and refractory residue materials are on the order of those appropriate for other refractory solids $\left(10^{-1}\right.$ to $10^{-3}$ atoms $/ 100-\mathrm{keV}$ proton) (Andersen and Bay 1976, Betz and Wehner 1983). At the fluences associated with the solar wind ions, much of the carbon is removed, leaving a small carbonized layer on a refractory core. It has been also suggested that, as observed in the laboratory, ion irradiation can strongly enhance the adhesion between silicate particles, core-mantle particles, and organic material, thus contributing to the formation of stable, fluffy aggregates possibly similar to Brownlee particles (Strazzulla et al. 1985, Johnson 1985). It has also been shown that the IR spectra of residues from ion-irradiated ices (see Figure 4) show features very similar to those from some meteorite extracts (Strazzulla et al. 1985).

In addition to the above, densification occurs. In Figure 14, the relative density variation is reported versus time for organic material on or in IDPs supposedly released by comets at 1 to $2 \mathrm{AU}$. The results are scaled from those in Figure 7 and refer to flux of the present solar wind for 1-keV H${ }^{+}$and 50-keV Fe (e.g., Jull et al. 1980) and solar flares for 100-keV H+ (e.g., Lanzerotti et al. 1978). Since the range of 1-keV/a.m.u. ions in the solid material in a cometary grain is $\sim 0.1$ to $0.005 \mu \mathrm{m}$, the results in Figure 14 apply to small particles. As a large fraction of IDPs consists of a fluffy agglomeration of submi- 


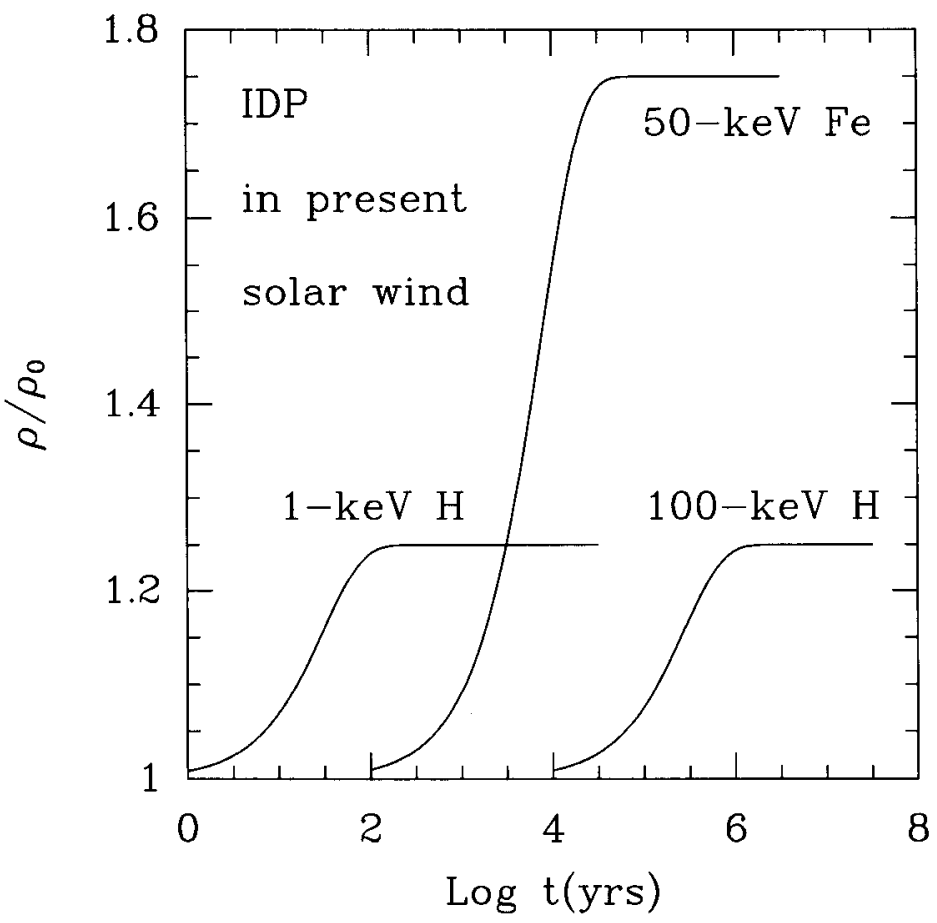

Figure 14. The densification of the organic content in IDPs due to irradiation of $\sim$ 1-keV/a.m.u. solar wind $\mathrm{H}$ and $\mathrm{Fe}$ ions and 100-keV flare $\mathrm{H}$ ions is shown versus time.

crometer particles with a very large area/volume ratio, most of the particles in the IDP are struck by solar wind ions. From Figure 14, we can see that in $10^{4}$ to $10^{5} \mathrm{yr}$, the time an IDP spends before collection, a significant increase in the density occurs. This process could help in explaining the time evolution of the physical parameters of IDPs inferred from observations (e.g., Le Sergeant d'Hendecourt and Lamy 1980, Fechtig and Mukai 1985).

\section{Conclusions}

The presence of organics in cometary materials and the fact that a large fraction of the carbon in a comet is in a refractory form suggest that irradiation processing of small molecules has occurred. Since surfaces (through heterogeneous catalysis) can enhance such processing and small grains are known to be present, this processing may have occurred while the molecular species were on the grains. Although nonlinear processes such as discharges in condensed (Khare et al. 1984) or in gas-phase (Sack et al. 1988) molecular mixtures can also produce linked carbon, we have reviewed here the nature and scale of those alterations induced by low-flux particle irradiations of the condensed state. It is seen that such irradiations occur in at least four separate phases in the evolution of 
cometary material from its existence in the interstellar medium until it is finally collected at or near the Earth.

In the interstellar medium, UV photo processing of materials is likely to dominate, although cosmic-ray ions always produce nonnegligible doses even in so-called "dark" molecular clouds. In the latter case, the contributing photons are primarily produced by the cosmic-ray energy loss in the ambient neutral gas, presuming there is more total material (by mass) as neutral gas-phase species than in the condensed state. Since the ratio of condensed to gas-phase molecules also may be determined by heavy cosmic-ray nuclei (Leger et al. 1985), the ultimate source of conversion of small molecules to more refractory solids is likely to be cosmic-ray ions.

During the collapse of a "molecular" cloud to form a sun, volatiles may be removed and/or reprocessed in the inner solar system by high temperatures, flares, etc. In the distant solar system, the interstellar grains and/or any newly formed material can accrete. The subsequent T-Tau phase not only removes gases and small grains from the inner solar system (Harris 1978), but also destroys small grains and processes the surfaces $\left(\simeq 10 \mathrm{~g} / \mathrm{cm}^{2}\right)$ of large aggregates or grains or cometesimals at distances $\leq 100$ AU. Subsequent accretion produces the larger objects dynamically expelled to the Oort cloud. These are continuously exposed to the cosmic-ray flux, producing a primordial refractory mantle $\left(\cong 30 \mathrm{~g} / \mathrm{cm}^{2}\right)$ on the comet and an altered mantle of volatiles $\left(\cong 300 \mathrm{~g} / \mathrm{cm}^{2}\right)$.

The presence of modified outer layers is suggested by a number of observations on new comets: "enhanced" activity at large distances, the recent finding of an altered ortho-topara ratio in $\mathrm{H}_{2} \mathrm{O}$, and possibly the observation of ejected $\mathrm{S}_{2}$ from the nucleus of a new comet. There has been speculation as to whether or not this "crust" (mantle) is lost, requiring new mantle formation processes (e.g., Rickman and Fernandez 1986). It is our contention, however, that the primordial crust is "lost" from the comet in only a few unstable places, giving rise to the enhanced activity on the first pass. These become the active regions and, of course, new active regions may be formed by fracturing or by impacts that pierce the crust (e.g., Rickman 1987). The primordial crust "grows" somewhat by the subsequent accretion processes occurring in the inner Solar System (e.g., Prialnik and Barnun 1988), and such processes may also control the behavior of the active regions.

Because a thick comet "crust" is likely, a comet sample return mission must first explore the surface to find regions with thin "crusts."

Because radicals produced by irradiation can be stored for long periods of time, there will be additional activity on the first pass when the heat pulse reaches a region of altered ices, as pointed out by Donn (1976). As subsequent irradiation processing of the active regions of the nucleus is small on each pass (Johnson et al. 1983), the "final" charged-particle irradiation effects occur when the ejected material is exposed to the solar wind and solar flares. This processing produces IDPs that have considerable tensile strength and have thin outer layers of highly processed (linked) carbon.

In order to quantify the important processes described here, as well as those occurring due to UV irradiation, it is no longer sufficient to simply note comparisons between observed spectral features and laboratory features. Rather, it is important to improve the description of the accretion processes and then carry out experiments that quantify particular material alteration processes, taking care to eliminate nonlinear (heating, diffusion, etc.) effects. 


\section{Acknowledgements}

GS is grateful to the Italian Space Agency (ASI) for financial support. REJ acknowledges NASA grant NAGW-186 and the NSF Astronomy division under grant AST-88-17700.

\section{References}

Allen, D.A., and Wickramasinghe, D.T. (1987). 'Discovery of organic grains in comet Wilson.' Nature 329, 615-616.

Andersen, H.H., and Bay, H.L. (1981). 'Sputtering yield measurements.' In Sputtering by Particle Bombardment I, R. Behrisch (ed.), Springer-Verlag, Berlin, pp. 145-218.

Andronico, G., Baratta, G.A., Spinella, F., and Strazzulla, G. (1987). 'Optical evolution of laboratory-produced organics: Applications to Phoebe, Iapetus, outer belt asteroids and cometary nuclei.' Astron. Astrophys. 184, 333-336.

Baas, F., Geballe, T.R., and Walther, D.M. (1986). 'Spectroscopy of the 3.4 micron emission feature in comet Halley.' Astrophys. J. Lett. 311, L97-L101.

Betz, G., and Wehner, G.K. (1983). 'Sputtering of multicomponent materials.' In Sputtering by Particle Bombardment II, R. Behrisch (ed.), Springer-Verlag, Berlin, pp. 11-90.

Boring, J.W., Nansheng, Z., Chrisey, D.B., O'Shaughnessey, D.J., Phipps, J.A., and Johnson, R.E. (1985). 'The production of $S_{2}$ by keV ion bombardment.' In Asteroids, Comets and Meteors II, C.I. Lagerkvist, B.A. Lindblad, H. Lundstedt, and H. Rickman (eds.), University of Uppsala Press, Uppsala, pp. 229-234.

Bradley, J.P., Brownlee, D.E., and Fraundorf, P. (1984). 'Discovery of nuclear tracks in interplanetary dust.' Science 226, 1432-1434.

Bregman, J.D., et al. (1987). 'Airborne and ground based spectrophotometry of comet P/Halley from 5-13 micrometers.' Astron. Astrophys. 187, 616-620.

Brown, W.L., and Johnson, R.E. (1986). 'Sputtering of ices: A review.' Nucl. Instr. and Meth. B13, 295-303.

Brown, W.L., Augustyniak, W.M., Lanzerotti, L.J., Johnson, R.E., and Evatt, R. (1980). 'Linear and non linear processes in the erosion of $\mathrm{H}_{2} \mathrm{O}$ ice by fast light ions.' Phys. Rev. Lett. 45, 1632-1635.

Brown, W.L., Lanzerotti, L.J., and Johnson, R.E. (1982a). 'Fast ion bombardment of ices and its astrophysical implications.' Science 218, 525-531.

Brown, W.L., Augustyniak, W.M., Simmons, E., Marcantonio, K.J., Lanzerotti, L.J., Johnson, R.E., Boring, J.W., Reimann, C.T., Foti, G., and Pirronello, V. (1982b). 'Erosion and molecular formation in condensed gas films by electronic energy loss of fast ions.' Nucl. Instr. Methods 198, 1-8.

Brown, W.L., Foti, G., Lanzerotti, L.J., Bower, J.E., and Johnson R.E. (1987). 'Delayed emission of hydrogen from ion bombardment of solid methane.' Nucl. Instr. Methods B19/20, 899-902.

Chrisey, D.B., Boring, J.W., Johnson, R.E., and Phipps, J.A. (1988). 'Molecular ejection from low temperature sulfur by keV ions.' Surf. Sci. 195, 594-618.

Chrisey, D.B., Brown, W.L., and Boring J.W. (1989). 'Electronic excitation of condensed CO: Sputtering and chemical change.' Surf. Sci., in press. 
Chyba, C., and Sagan, C. (1987). 'Infrared emission by organic grains in the coma of comet Halley.' Nature 330, 350-353.

Chyba, C., and Sagan, C. (1988). 'Cometary organic matter still a contentious issue.' Nature 332, 592.

Combes, M., et al. (1986). 'Infrared sounding of comet Halley from Vega 1.' Nature $321,266-268$.

Combi, M.R. (1987). 'Sources of cometary radicals and their jets: Gases or grains.' Icarus 71, 178-191.

Cosmovici, C., and Ortolani, S. (1984). 'Detection of new molecules in the visible spectrum of comet IRAS-Araki-Alcock (1984).' Nature 310, 122-124.

de Jong, T., and Kamijo F., (1973). 'Growth and destruction of interstellar grains in the presence of low-energy cosmic rays.' Astron. Astrophys. 25, 363-370.

Donn, B. (1976). 'The nucleus: Panel discussion.' In The Study of Comets, B. Donn et al. (eds.), NASA SP-393, pp. 611-621.

Donn, B., and Hughes, D. (1986). 'A fractal model of a cometary nucleus formed by random accretion.' ESA SP-250, vol. III, pp. 523-524.

Draganic, I.G., Draganic, Z.D., and Vujosevic, S.I. (1984). 'Some radiation-chemical aspects of chemistry in cometary nuclei.' Icarus $60,464-475$.

Fechtig, H., and Mukai, T. (1985). 'Dust of variable porosities (densities) in the Solar System.' In Ices in the Solar System, J. Klinger et al. (eds.), D. Reidel Publ. Co., Dordrecht, pp. 251-259.

Feigelson, E.D. (1982). 'X-ray emission from young stars and implications for the early Solar System.' Icarus 51, 155-163.

Field, G.B., Goldsmith, D.W., and Habing, H.J. (1969). 'Cosmic-ray heating of interstellar gas.' Astrophys. J. Lett. 155, L149-L154.

Foti, G., Calcagno, L., Sheng, K.L., and Strazzulla, G. (1984). 'Micrometre-sized polymer layers synthesized by $\mathrm{MeV}$ ions impinging on frozen methane.' Nature 310 , 126-128.

Foti, G., Calcagno, L., Zhu, F.Z., and Strazzulla, G. (1987). 'Chemical evolution of solid methane by keV ion bombardment.' Nucl. Instr. Methods in Phys. Res. B24-25, $522-525$.

Gombosi, T.I., and Houpis, H.L.F. (1986). 'An icy-glue model of cometary nuclei.' Nature 324, 43-44.

Greenberg, J.M. (1982). 'What are comets made of? A model based on interstellar dust.' In Comets, L.L. Wilkening (ed.), University of Arizona Press, Tucson, pp. 131-163.

Greenberg, J.M., and Zhao, N. (1988). 'Cometary organics.' Nature 331, 124.

Hanner, M.S., Knacke, R., Sekanina, Z., and Tokunaga, A.T. (1985). 'Dark grains in comet Crommelin.' Astron. Astrophys. 152, 177-181.

Haring, R.A., Haring A., Klein, F.S., Kummel, A.C., and de Vries, A.E. (1983). 'Reactive sputtering of simple condensed gases by keV heavy ion bombardment.' Nucl. Instrum. Methods 211, 529-538.

Harris, A.W. (1978). 'Dynamics of planetesimals formation and planetary accretion.' In the Origin of the Solar System, S.F. Dermott (ed.), Wiley, New York, pp. 469-492.

Hart, E.J., and Platzman, R.L. (1961). 'Radiation chemistry' In Physical Mechanisms in Radiation Biology 1, Academic Press, New York, pp. 93-120.

Hoyle, F., and Wickramasinghe, N.C. (1985). Living Comets. University College Press, London. 
Hoyle, F., and Wickramasinghe, N.C. (1987). 'Organic dust in comet Halley.' Nature $328,117$.

Huebner, W.F. (1987). 'First polymer in space identified in Comet Halley.' Science 237, $628-630$.

Jessberger, E.K., Cristoforidis, A., and Kissel, J. (1988). 'Aspects of the major element composition of Halley's dust.' Nature 332, 691-695.

Johnson, R.E. (1985). 'Comment on the evolution of interplanetary grains.' In Ices in the Solar System, J. Klinger et al. (eds.), D. Reidel Publ. Co., Dordrecht, pp. 337-339.

Johnson, R.E. (1989a). Energetic Charged-Particle Interactions With Atmospheres and Surfaces. Springer-Verlag, Berlin, in press.

Johnson, R.E. (1989b). 'Laboratory simulations: The primordial comet mantle.' In the NASA publication for the Comet Sample Return Conference, Milipitas, 1989, in press.

Johnson, R.E., and Lanzerotti, L.J. (1986). 'Ion bombardment of interplanetary dust.' Icarus 66, 619-624.

Johnson, R.E., Lanzerotti, L.J., Brown, W.L., Augustyniak, W.M., and Mussil, C. (1983). 'Charged particle erosion of frozen volatiles in ice grains and comets.' Astron. Astrophys. 123, 343-346.

Johnson, R.E., Lanzerotti, L.J., and Brown, W.L. (1984). 'Sputtering processes: Erosion and chemical change.' Adv. Space Res. 4, 41-51.

Johnson, R.E., Barton, L.A., Boring, J.W., Jesser, W.A., Brown, W.L., and Lanzerotti, L.J. (1985). 'Charged particle modification of ices in the Jovian and Saturnian systems.' In Ices in the Solar System, J. Klinger et al. (eds.), D. Reidel Publ. Co., Dordrecht, pp. 301-316.

Johnson, R.E., Cooper, J.F., Lanzerotti, L.J., and Strazzulla, G. (1987). 'Radiation formation of a non-volatile comet crust.' Astron. Astrophys. 187, 889-892.

Jull, A.J.T., Wilson, G.C., Long, J.V.P., Reed, S.J.B., and Pillinger, C.T. (1980). 'Sputtering rates of minerals and implications for abundances of solar elements in lunar samples.' Nucl. Instr. Methods 168, 357-365.

Khare, B.N., Sagan, C., Arakawa, E.T., Suits, F., Calcott, T.A., and Williams, M.W. (1984). 'Optical constants of organic tholins produced in a simulated Titanian atmosphere from X-ray to microwave frequencies.' Icarus 60, 127-137.

Kissel, J., and Krueger, F.R. (1987a). 'The organic component in dust from comet Halley as measured by the PUMA mass spectrometer on board Vega 1.' Nature 326, 755-760.

Kissel, J., and Krueger, F.R. (1987b). 'Organic dust in comet Halley.' Nature 328, 117.

Kissel, J., et al. (1986a). 'Composition of comet Halley dust particles from Vega observations.' Nature 321, 280-282.

Kissel, J., et al. (1986b). 'Composition of comet Halley dust particles from Giotto observations.' Nature 321, 336-337

Knacke, R.F., Brooke, T.Y., and Joyce, R.R. (1987). 'The 3.2-3.6 $\mu \mathrm{m}$ emission features in comet P/Halley: Spectral identification and similarities.' Astron. Astrophys. $187,625-628$.

Lanzerotti, L.J., Brown, W.L., Poate, C.M., and Augustyniak, W.M. (1978). 'Low energy cosmic ray erosion of ice grains in interplanetary and interstellar media.' Nature 272, 431-433. 
Lanzerotti, L.J., Brown, W.L., and Johnson, R.E. (1985). 'Laboratory studies of ion irradiation of water, sulfur dioxide, and methane ices.' In Ices in the Solar System, J. Klinger et al. (eds.), D. Reidel Publ. Co., Dordrecht, pp. 317-333.

Lanzerotti, L.J., Brown, W.L., and Marcantonio, K.J. (1987). 'Experimental study of erosion of methane ice by energetic ions and some considerations for astrophysics.' Astrophys. J. 313, 910-922.

Leger, A., Jura, M., and Amont, A. (1985). 'Desorption from interstellar grains.' Astron. Astrophys. 144, 147-160.

Le Sergeant d'Hendecourt, L.B., and Lamy, Ph. L. (1980). 'On the size distribution and physical properties of interplanetary dust grains.' Icarus 43, 350-372.

Littmark, U., and Ziegler, J.F. (1980). Handbook of Range Distributions for Energetic Ions in All Elements. Pergamon, Elmsford, New York.

Mazzoldi, P., and Arnold, G.W. (1987). Ion Beam Modification of Insulators. Elsevier, Amsterdam.

Melcher, C.L., LePoire, D.J., Cooper, B.H., and Trombello, T.A. (1982). 'Erosion of frozen sulfur dioxide by ion bombardment: Application to Io.' Geophys. Res. Lett. 9, 1151-1154.

Mitchell, D.L. et al. (1987). 'Evidence for chain molecules enriched in carbon, hydrogen and oxygen in comet Halley.' Science 237, 626-628.

Moore, M.H. (1982). 'Studies of proton-irradiated cometary-type ice mixtures.' Ph.D. thesis, University of Maryland, College Park, Maryland.

Moore, M.H., and Donn, B. (1982). 'The infrared spectrum of a laboratory synthesized residue: Implication for the $3.4 \mu \mathrm{m}$ interstellar absorption feature.' Astrophys. J. 257, L47-L50.

Moore, M.H., Donn, B., Khanna, R., and A'Hearn, M.F. (1983). 'Studies of protonirradiated cometary-type ice mixtures.' Icarus 54, 388-405.

Moroz, V.I., et al. (1987). 'Detection of parent molecules in comet P/Halley from IKSVega experiment.' Astron. Astrophys. 187, 513-518.

Mukai, T., and Schwehm, G. (1981). 'Interaction of grains with the solar energetic particles.' Astron. Astrophys. 95, 373-382.

Mumma, M.J., Blass, W.E., Weaver, H.A., and Larson, H.P. (1989). 'Measurements of the ortho-para ratio and the nuclear spin temperatures of water vapour in comet Halley and Wilson (1986i) and the implication for their origin and evolution.' Submitted.

O'Shaughnessy, D.J., Boring, J.W., Philipps, J.A., Johnson, R.E., and Brown, W.L. (1986). 'Sputtering of rare gas solids by keV ions.' Nucl. Instr. and Meth. B13, 304 308.

Pirronello, V., and Lanzafame, G. (1989). 'Molecules synthesized by cosmic rays in cometary nuclei: A tool to estimate their low energy spectrum.' Astrophys. J., in press.

Pirronello, V., Brown, W.L., Lanzerotti, L.J., Marcantonio, K.J., and Simmons, E. (1982). 'Formaldehyde formation in a $\mathrm{H}_{2} \mathrm{O} / \mathrm{CO}_{2}$ ice mixture under irradiation by fast ions.' Astrophys. J. 262, 636-640.

Pirronello, V., Strazzulla, G., Foti, G., Brown, W.L., and Lanzerotti, L.J. (1984). 'Formaldehyde formation in cometary nuclei.' Astron. Astrophys. 134, 204-206.

Prialnik, D., and Bar-Nun, A. (1988). 'The formation of a permanent dust mantle and its effect on cometary activity.' Icarus 74, 272-283. 
Reimann, C.T., Boring, J.W., Johnson, R.E., Garret, J.W., Farmer, K.R. and Brown, W.L. (1984). 'Ion-induced molecular ejection from $\mathrm{D}_{2} \mathrm{O}$ ice.' Surf. Sci. 147, 227240.

Rickman, H. (1986). 'Masses and densities of comets Halley and Kopff.' In The Comet Nucleus Sample Return Mission, ESA SP-249, pp. 195-205.

Rickman, H. (1987). 'Physical evolution of comets.' In Proc. 10th European Astronomy Meeting of the IAU, Prague 1987.

Rickman, H., and Fernandez, J.A. (1986). 'Formation and blowoff of a cometary dust mantle.' In The Comet Nucleus Sample Return Mission, ESA SP-249, pp. 185-195.

Ryan, M.P., Jr., and Draganic, I.G. (1986). 'An estimate of the contribution of high energy cosmic-ray protons to the absorbed dose inventory of a cometary nucleus.' Astrophys. Space. Sci. 125, 49-68.

Sack, N., Schuster, R., Hoffman, A., and Schneider, H.J. (1988). 'Production of amines by proton bombardment of simple condensed gases.' Icarus 76, 110-117.

Sagdaeev, R.Z., Elyasberg, P.E., and Moroz, V.I. (1988). 'Is the nucleus of comet Halley a low density body?' Nature 331, 240-242.

Stern, S.A. (1988). 'Collisions in the Oort cloud.' Icarus 73, 499-507.

Stern, S.A., and Shull, M.J. (1988). 'The relevance of supernovas and passing stars on comets.' Nature 332, 407-411.

Strazzulla, G. (1985). 'Modifications of grains by particle bombardment in the early Solar System.' Icarus 61, 48-56.

Strazzulla, G. (1986). “'Primitive" galactic dust in the Solar System?' Icarus 67, 63-70.

Strazzulla, G. (1988). 'Ion bombardment: Techniques, materials and applications.' In Experiments on Cosmic Dust Analogues, E. Bussoletti et al. (eds.), Kluwer Acad. Publ., Dordrecht, pp. 103-113.

Strazzulla, G., Calcagno, L., and Foti, G. (1983a). 'Polymerization induced on interstellar grains by low energy cosmic rays.' M.N.R.A.S. 204, 59p-62p.

Strazzulla, G., Pirronello, V., and Foti, G. (1983b). 'Physical and chemical effects induced by energetic ions on comets.' Astron. Astrophys. 123, 93-97.

Strazzulla, G., Calcagno, L., and Foti, G. (1984). 'Build up of carbonaceous material by fast protons on Pluto and Triton.' Astron. Astrophys. 140, 441-444.

Strazzulla, G., Calcagno, L., Foti, G., and Sheng, K.L. (1985). 'Interaction between solar energetic particles and interplanetary grains.' In Ices in the Solar System, J. Klinger et al. (eds.), D. Reidel Publ. Co., Dordrecht, pp. 73-285.

Strazzulla, G., Torrisi, L., and Foti, G. (1988). 'Light scattering from ion irradiated frozen gases.' Europhys Lett. 7(5), 431-434.

Tokunaga, A.T., Nagata, T., and Smith, R.G. (1987). 'Detection of a new emission band at $2.8 \mu \mathrm{m}$ in comet P/Halley.' Astron. Astrophys. 187, 519-522.

Torrisi, L., Coffa, S., Foti, G., and Strazzulla, G. (1986). 'Sulphur erosion by $1.0 \mathrm{MeV}$ helium ions.' Radiation Eff. 100, 61-69

Torrisi, L., Coffa, S., Foti, G., Johnson, R.E., Chrisey, D.B., and Boring, J.W. (1988). 'Threshold dependence in the electronic sputtering of condensed sulfur.' Phys. Rev. B 38, 1516-1519.

Vanysek, M.K., and Wickramasinghe, N.C. (1975). 'Formaldehyde polymers in comets.' Astrophys. Space Sci. 33, L19-L28. 
Watson, W.D. (1975). 'Physical processes for the formation and destruction of interstellar molecules.' In Atomic and Molecular Physics and the Interstellar Matter, R. Balian et al. (eds.), North Holland Publ. Co., Amsterdam, pp. 177-271.

Weissman, P.R. (1986). 'Are cometary nuclei primordial rubble piles?' Nature 320, 242244.

Whipple, F.L. (1950). 'A comet model: The acceleration of comet Encke.' Astrophys. J. $111,375-394$.

Whipple, F.L. (1972). 'The origin of comets.' In The Motion, Evolution of Orbits and Origin of Comets, IAU Symp. 45, D. Reidel Publ. Co., Dordrecht, pp. 401-408.

Whipple, F.L. (1977). 'The constitution of cometary nuclei.' In Comets, Asteroids, Meteorites: Interrelations, Evolution and Origins, A.H. Delsemme (ed.), University of Toledo, Toledo, Ohio, pp. 25-32.

Wickramasinghe, D.T., and Allen, D.A. (1986). 'Discovery of organic grains in comet Halley.' Nature 323, 44-46.

Wood, J.A. (1986). 'Comet nucleus models: A review.' In The Comet Nucleus Sample Return Mission, ESA SP-249, pp. 123-131.

Worden, S.P., Schneeberger, T.J., Kuhn, J.R., and Africano, J.L. (1981). 'Flare activity of T Tauri stars.' Astrophys. J. 244, 520-527.

Yamamoto, T., Nakagawa, N., and Fukui, Y. (1983). 'The chemical composition and thermal history of the ice of a cometary nucleus.' Astron. Astrophys. 122, 171-176. 